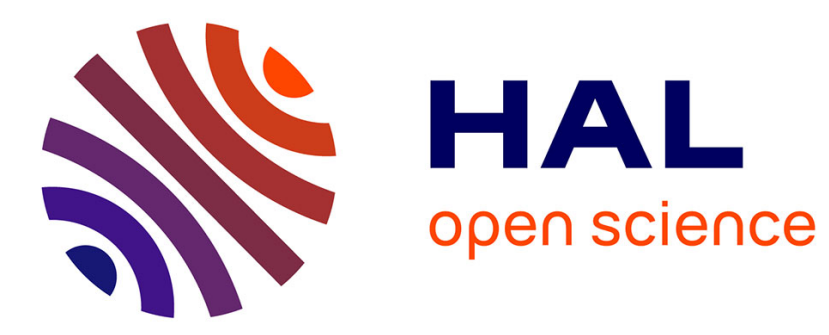

\title{
On supermatrix models, Poisson geometry and noncommutative supersymmetric gauge theories
}

Ctirad Klimcik

\section{To cite this version:}

Ctirad Klimcik. On supermatrix models, Poisson geometry and noncommutative supersymmetric gauge theories. Journal of Mathematical Physics, 2015, 56, pp.122302. 10.1063/1.4937450 . hal01246189

\author{
HAL Id: hal-01246189 \\ https://hal.science/hal-01246189
}

Submitted on 18 Dec 2015

HAL is a multi-disciplinary open access archive for the deposit and dissemination of scientific research documents, whether they are published or not. The documents may come from teaching and research institutions in France or abroad, or from public or private research centers.
L'archive ouverte pluridisciplinaire HAL, est destinée au dépôt et à la diffusion de documents scientifiques de niveau recherche, publiés ou non, émanant des établissements d'enseignement et de recherche français ou étrangers, des laboratoires publics ou privés. 


\section{A|P| $\left.\right|_{\text {Mathematical Physics }} ^{\text {Journal of }}$}

On supermatrix models, Poisson geometry, and noncommutative

\section{supersymmetric gauge theories}

Ctirad Klimčík

Citation: Journal of Mathematical Physics 56, 122302 (2015); doi: 10.1063/1.4937450

View online: http://dx.doi.org/10.1063/1.4937450

View Table of Contents: http://scitation.aip.org/content/aip/journal/jmp/56/12?ver=pdfcov

Published by the AIP Publishing

\section{Articles you may be interested in}

Gauge theories in noncommutative homogeneous Kähler manifolds

J. Math. Phys. 55, 092301 (2014); 10.1063/1.4893982

Noncommutative gauge supersymmetric theory in two dimensions in Minkowski space AIP Conf. Proc. 1444, 434 (2012); 10.1063/1.4715471

Gauge theories in noncommutative geometry

AIP Conf. Proc. 1446, 73 (2012); 10.1063/1.4727990

Consistent anomalies in translation-invariant noncommutative gauge theories

J. Math. Phys. 53, 042303 (2012); 10.1063/1.4704797

Non-Abelian gauge field theory in scale relativity

J. Math. Phys. 47, 032303 (2006); 10.1063/1.2176915

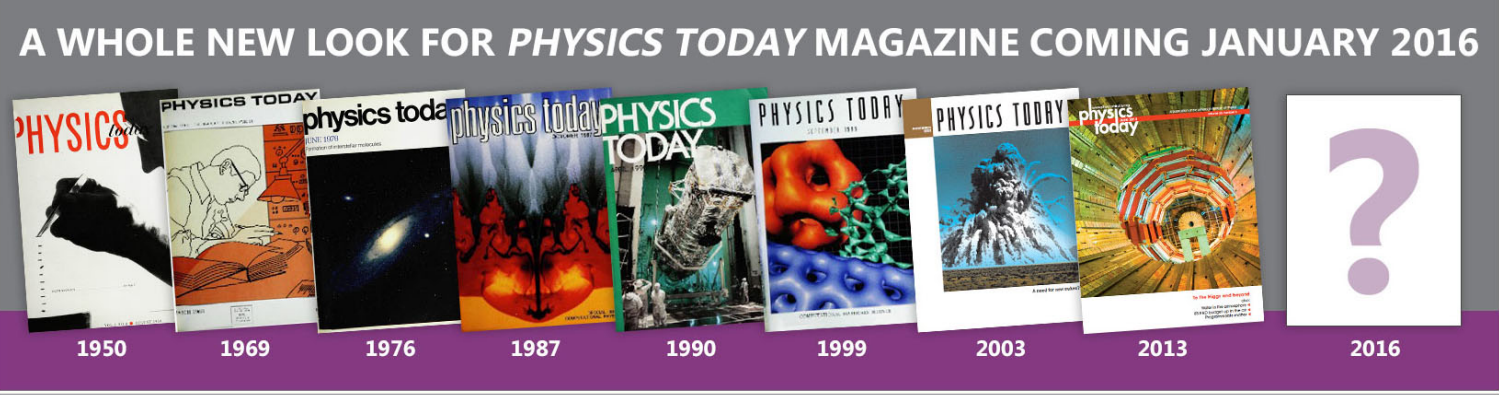




\title{
On supermatrix models, Poisson geometry, and noncommutative supersymmetric gauge theories
}

\author{
Ctirad Klimčík \\ Aix Marseille Université, CNRS, Centrale Marseille I2M, UMR 7373, \\ 13453 Marseille, France
}

(Received 22 May 2015; accepted 23 November 2015; published online 16 December 2015)

\begin{abstract}
We construct a new supermatrix model which represents a manifestly supersymmetric noncommutative regularisation of the $\operatorname{UOSp}(2 \mid 1)$ supersymmetric Schwinger model on the supersphere. Our construction is much simpler than those already existing in the literature and it was found by using Poisson geometry in a substantial way. (C) 2015 AIP Publishing LLC. [http://dx.doi.org/10.1063/1.4937450]
\end{abstract}

\section{INTRODUCTION}

The subject of this paper is situated on a crossroad of two active current themes of research: the first concerns the application of the method of localisation ${ }^{28}$ to extract quantitative information about rigidly supersymmetric Euclidean field theories on compact manifolds and the second deals with the study of (super)matrix models giving rise to noncommutative field theories with various amount of (super)symmetry. It is certainly impossible to provide a complete bibliography of relevant works in both directions we can however mention Refs. 2, 3, 6, 8, 7, 10-13, 17, 16, 30,32 , and 35, respectively, 4, 20, 19, 18, 24, 33, and 36 which treat the gauge theories living on two-dimensional compact Euclidean (super)spaces, mostly on a sphere $S^{2}$ or on a supersphere $S^{2 \mid 2}$.

Probably, the first example of a supersymmetric gauge theory on the supersphere was defined and studied in Ref. 25, namely, the $\operatorname{UOSP}(2,1)$ invariant supersymmetric extension of the standard Schwinger model on $S^{2}{ }^{31,21}$ This rigidly supersymmetric model yields in the decompactification limit the minimal $N=(1,1)$ supersymmetric electrodynamics on the plane constructed already by Ferrara in Ref. 9 and it was studied in Ref. 25 with the main motivation to find its manifestly supersymmetric noncommutative regularisation keeping only a finite number of degrees of freedom in the theory. This goal was indeed achieved in Ref. 25 and the resulting theory even flaunted a solid geometrical status in both commutative and noncommutative versions. In particular, it allowed also a formulation in terms of a supermatrix model as discovered later in Refs. 19 and 18. In spite of all of those successes, here we come back to the subject to present another construction which accomplishes precisely the same objectives as Ref. 25 but is at the same time much simpler. The basic ingredient making possible to simplify the story of Ref. 25 is the use of Poisson geometry which not only allows to guess natural candidates for $\operatorname{uosp}(2 \mid 1)$ supersymmetric gauge invariant Lagrangians but it also incredibly streamlines and speeds up the technical work needed to verify that they have the required properties. We invented and tested this new Poisson formalism while studying supersymmetric $\sigma$-models ${ }^{26}$ and we are pleased to confirm its efficiency in the present work.

Let us thus straightaway write down two principal results of the present article. The first one is a new very simple version of the action principle of the $\operatorname{UOSp}(2 \mid 1)$ supersymmetric Schwinger model on the supersphere $S^{2 / 2}$. It reads

$$
S(\Phi, \mathcal{E})=\int d \mu_{S^{2 \mid 2}}\left(\left\|\left\{\mathcal{M}^{2}, \Phi\right\}+[\mathcal{M}, \mathcal{E}] \Phi\right\|^{2}+\frac{1}{4 e^{2}}\left\|\left\{\mathcal{M}^{2}, \operatorname{str}\left\{\mathcal{M}^{2}, \mathcal{E}\right\}\right\}\right\|^{2}\right) .
$$

The second one is a new action of the supermatrix model which in the limit of the infinite size of the supermatrices yields theory (1.1). It is given by 


$$
S_{N}(\hat{\Phi}, \mathcal{P})=\alpha_{N} \operatorname{STr} \operatorname{str}\left((\mathcal{P} \hat{\Phi}-\hat{\Phi} \hat{\mathcal{K}})^{\dagger}(\mathcal{P} \hat{\Phi}-\hat{\Phi} \hat{\mathcal{K}})-\frac{\sigma_{N}^{2}}{e^{2}}\left[\mathcal{P}, \operatorname{str}\left(\mathcal{P}_{v}^{2} \mathcal{P}_{v}^{2}-\hat{\mathcal{K}}_{v}^{2} \hat{\mathcal{K}}_{v}^{2}\right)\right]^{2}\right),
$$

where the $N$-dependence of the constants $\alpha_{N}$ and $\sigma_{N}$ is made explicit in (3.85) and (3.86).

The Introduction is not a place where all technical details should be given; nonetheless, we believe that it is helpful to provide the reader with a rough acquaintance with the notations met in (1.1) and (1.2) already at this level. Thus, $\Phi$ stands for the charged matter superfield on $S^{2 / 2}$ and the $(N+1 \mid N) \times(N+1 \mid N)$ supermatrix $\hat{\Phi}$ is its noncommutative analogue. $\mathcal{E}$ is the so-called superspinorial $(2 \mid 1) \times(2 \mid 1)$ supermatrix the entries of which are functions on $S^{2 / 2}$ and it plays the role of the gauge superfield on the supersphere. $\mathcal{P}$ is the noncommutative analogue of $\mathcal{E}$, it is the superspinorial $(2 \mid 1) \times(2 \mid 1)$ supermatrix the entries of which are $(N+1 \mid N) \times(N+1 \mid N)$ supermatrices. The bracket $\{.,$.$\} is the Kirillov-Costant-Souriau Poisson bracket { }^{37}$ on $S^{2 / 2}$ and the supervectorial $(2 \mid 1) \times(2 \mid 1)$ supermatrix $\mathcal{M}$ is the moment map generating the infinitesimal $\operatorname{uos} p(2 \mid 1)$ transformations of $S^{2 \mid 2}$ via these Poisson brackets. Finally, $\hat{\mathcal{K}}$ is the noncommutative analogue of $\mathcal{M}^{2}$.

It must be stressed that the compact notation appearing in (1.1) and (1.2) was not conceived forcibly but it is very natural. We mean by this that one can perform nontrivial technical operations on our actions working directly in the succinct notation without choosing a basis of the Lie superalgebra $\operatorname{uos} p(2 \mid 1)$, without detailing the entries of the moment map $\mathcal{M}$ or of the superspinorial supermatrix $\mathcal{E}$, and, of course, without expanding the superfields in components. In particular, the $\operatorname{uosp}(2 \mid 1)$ superinvariance as well as the gauge invariance of action (1.1) can be checked in this concise way just by making use of some basic properties of the Poisson brackets like the Jacobi identity. For that matter, we believe that the use of the Poisson geometry in the construction of the supersymmetric invariants will prove to be useful also for other compact supermanifolds enjoying rigid supersymmetry whenever the action of the corresponding Lie superalgebra on the supermanifold is Hamiltonian.

The plan of the paper is as follows: in Section II, we first present purely bosonic counterparts (2.29) and (2.62) of main results (1.1) and (1.2). In Section III A, we review the basic properties of the supermatrices and of the supersphere; in Section III B, we construct the action (1.1) and establish its symmetry properties; and in Section III C, we show that the expansion of supersymmetric action (1.1) in components contains purely bosonic action (2.29) as well as the standard Schwinger model on the sphere. ${ }^{31,21}$ We review the concept of the fuzzy supersphere in Section III D and in Section III E, we finally construct supermatrix model (1.2), we establish its symmetry properties, and we prove that in the large $N$ limit it yields supersymmetric gauge theory (1.1). Section IV is devoted to a discussion of the results. It should also be read carefully since we formulate there some interesting geometrical questions that our concept of the supergauge field poses.

\section{BOSONIC WARM UP}

\section{A. Manifestly so(3) symmetric electrodynamics on $s^{2}$}

The standard way for writing down the action of a scalar electrodynamics living on a twodimensional Riemannian space-time $M$ with Euclidean signature uses the determinant $\sqrt{g}$ of the Riemann tensor $g_{\mu \nu}$ and the components $g^{\mu \nu}$ of it inverse,

$$
S\left(\phi, A_{\mu}\right)=\frac{1}{2} \int_{M} d^{2} \xi \sqrt{g}\left(g^{\mu \nu}\left(\partial_{\mu}+i A_{\mu}\right) \bar{\phi}\left(\partial_{\mu}-i A_{\mu}\right) \phi+\frac{1}{e^{2}} g^{\mu \rho} g^{v \sigma} F_{\mu \nu} F_{\rho \sigma}\right) .
$$

Here, $A_{\mu}$ is the electromagnetic potential in some coordinates $\xi^{\mu}, e^{2}$ is the coupling constant, and $F_{\mu \nu}$ is the field strength,

$$
F_{\mu \nu}=\partial_{\mu} A_{\nu}-\partial_{\nu} A_{\mu}
$$

Of course, a potential term $\int d \mu_{S^{2}} V(\bar{\phi} \phi)$ can be obviously added to this action but we shall be systematically avoiding it as our principal concern is the interaction of the matter field $\phi$ with the gauge field $A_{\mu}$. 
If the manifold $M$ is the unit two-sphere $S^{2}$ equipped with the standard round Riemannian metric, then action (2.1) can be rewritten ${ }^{38}$ in an $S O(3)$-covariant way as

$$
S\left(\phi, A_{k}\right)=\frac{1}{2} \int d \mu_{S^{2}}\left(\left(R_{k}+i A_{k}\right) \bar{\phi}\left(R_{k}-i A_{k}\right) \phi+\frac{1}{e^{2}} F_{k}(A) F_{k}(A)\right),
$$

where the $S O(3)$ covariant electromagnetic field strength vector $F_{k}$ is defined as

$$
F_{k}(A):=\epsilon_{k l m}\left(R_{l} A_{m}-R_{m} A_{l}+\epsilon_{l m p} A_{p}\right) .
$$

In order to explain the meaning of the symbols $A_{k}$ and $R_{k}, k=1,2,3$, we first need to introduce three functions $x_{1}, x_{2}, x_{3}$ on $S^{2}$ the values of which in every point of the sphere are given by the $\mathbb{R}^{3}$ Cartesian coordinates of this point (the unit sphere $S^{2}$ is thought to be standardly embedded in the three-dimensional Euclidean space $\mathbb{R}^{3}$ and the Riemannian metric on $S^{2}$ is induced from the flat one on $\mathbb{R}^{3}$ ). Thus, it holds

$$
x_{1}^{2}+x_{2}^{2}+x_{3}^{2}=1
$$

and the measure on $S^{2}$ induced by the round Riemannian metric can be written accordingly as

$$
d \mu_{S^{2}}=d x_{1} d x_{2} d x_{3} \delta\left(x_{1}^{2}+x_{2}^{2}+x_{3}^{2}-1\right) .
$$

The following vector fields $R_{k}$ on $\mathbb{R}^{3}$ are tangent to surface (2.5) of the embedded sphere; hence, they can be viewed also as the vector fields on $S^{2}$,

$$
R_{k}:=-\epsilon_{k l m} x_{l} \partial_{m}
$$

The vector fields $R_{k}$ generate an infinitesimal action of $S O(3)$ on $S^{2}$ and are related by an obvious identity following from the total antisymmetry of the Levi-Civita tensor $\epsilon_{k l m}$,

$$
x_{k} R_{k}=0 .
$$

To define $A_{k}$, we decompose the electromagnetic potential 1-form $A_{\mu} d \xi^{\mu}$ as

$$
A_{\mu} d \xi^{\mu}=A_{k} \beta_{k},
$$

where

$$
\beta_{k}:=-\epsilon_{k l m} x_{l} d x_{m} .
$$

If we furthermore impose a constraint

$$
A_{k} x_{k}=0,
$$

then the coefficient functions $A_{k}$ are determined uniquely from $A_{\mu} d \xi^{\mu}$ via (2.9) and (2.11). Constraint (2.11) has a natural geometric interpretation because it says that the $S O(3)$ vector $A_{k}$ is perpendicular to the normal vector $x_{k}$; hence, $A_{k}$ is tangent to the surface of the sphere.

Virtually, all authors working on the subject of the gauge theories on the fuzzy sphere $e^{1,4,20,23,24,22,33,34}$ used the manifestly $S O(3)$ invariant form (2.12) of the scalar electrodynamics on $S^{2}$ as the starting point to the construction of noncommutative deformations. It appears that nothing more can be said about (2.3), yet there is an almost "banal" detail which we remarked only recently and which actually triggered our renewed interest in the subject of the fuzzy deformations of (super)gauge field theories. The point is that action (2.3) can be rewritten in slightly different but still manifestly $S O(3)$-invariant way as follows:

$$
S\left(\phi, A_{k}\right)=\frac{1}{2} \int d \mu_{S^{2}}\left(\left(R_{k}+i A_{k}\right) \bar{\phi}\left(R_{k}-i A_{k}\right) \phi+\frac{1}{e^{2}} F(A)^{2}\right),
$$

where

$$
F(A):=\epsilon_{k l m} x_{k} R_{l} A_{m} .
$$

The quantity $F(A)$ can be referred to as a "scalar field strength" and it is invariant with respect to the $S O(3)$ transformations (infinitesimaly generated by the vector fields $R_{k}$ ) as well as with respect to the gauge transformations

$$
A_{k} \rightarrow A_{k}+R_{k} \rho
$$


Here, $\rho$ is arbitrary function on $S^{2}$. Notice also that gauge transformation (2.14) is compatible with constraint (2.11) due to (2.8).

Remark 1. In order to demonstrate the equivalence of actions (2.3) and (2.12), it is useful to parametrize the electromagnetic potential $A_{\mu} d \xi^{\mu}$ in terms of its Hodge dual $*\left(A_{\mu} d \xi^{\mu}\right)$,

$$
*\left(A_{\mu} d \xi^{\mu}\right):=B_{k} \beta_{k},
$$

where, as before, the coefficient functions $B_{k}$ are unambiguously fixed by the constraint

$$
B_{k} x_{k}=0 .
$$

It is easy to check that the relation between the respective $S O(3)$ covariant parametrizations $B_{k}$ of $*\left(A_{\mu} d \xi^{\mu}\right)$ and $A_{k}$ of $A_{\mu} d \xi^{\mu}$ reads

$$
B_{k}=-\epsilon_{k l m} x_{l} A_{m}, \quad A_{k}=\epsilon_{k l m} x_{l} B_{m} .
$$

By the way, it appears quite intriguing that the standard intrinsic two-dimensional Hodge star on the sphere $S^{2}$ has such an elegant formulation (2.17) when expressed in the manifestly $S O(3)$ invariant formalism essentially inherited from the embedding of the 2-sphere into the three-dimensional Euclidean space $\mathbb{R}^{3}$. We do not see a direct connection of this two-dimensional round Hodge star with the three dimensional Euclidean one but it would be certainly interesting in future to clarify this issue.

It is also easy to calculate the quantities $F_{k}$ and $F$ in the dual $B_{k}$ parametrization. The result is

$$
F_{k}=x_{k}\left(R_{m} B_{m}\right), \quad F=R_{m} B_{m},
$$

from which the equivalence of actions (2.3) and (2.12) readily follows.

Is there any conceptual or technical gain which could be extracted from rewriting (2.12) of action (2.3)? Well, as we shall see in Section II B, the noncommutative deformation based on new version (2.12) looks more or less as complicated as the standard one based on (2.3). However, the things are very different in the supersymmetric setting (cf. Section III B further on) where the existence of a scalar superfield strength generalizing the purely bosonic quantity $F$ turns out to simplify drastically the construction of the noncommutative supersymmetric gauge theory. The reason for this is the following: in the $\operatorname{UOSp}(2 \mid 1)$ supersymmetric analogue of action (2.3) constructed in Ref. 25, the role of the three-component field strength $F_{k}$ is played by an eighth-component object living in the adjoint representation of the supergroup $S U(2 \mid 1)$. This eight-component object has to be, furthermore, constrained in the $\operatorname{UOSp}(2 \mid 1)$ supersymmetric and supergauge invariant way. All in all, the already existing construction ${ }^{25}$ of the manifestly supersymmetric electrodynamics on the supersphere is quite involved while, as we shall see in Section III, it can be replaced by an astonishingly simple alternative, by using the scalar superfield strength as a "sesame."

\section{B. The use of Poisson geometry}

Before turning to the supersymmetric case, which is our real concern in this paper, we spend here some more time with the purely bosonic $S O(3)$ invariant gauge theory (2.12). We do it in order to illustrate the use of the new technical tools based on Poisson geometry. As we have seen in Section II A, in the purely bosonic case, the use of the Kronecker tensor and of the Levi-Civita tensor is sufficient to obtain all important formulas; therefore, the Poisson tools represent just an amusing computational alternative. However, in the supersymmetric case, the Poisson tools are considerably more beneficial from both conceptual and technical point of view because $s u(2 \mid 1)$ invariant tensors are more numerous than in $s o(3)$ case, they have more components and they are tied together by more complicated identities.

The Poisson geometry enters the game because there is a natural Kirillov-Kostant-Souriau Poisson bracket ${ }^{39}$ on $S^{2}$ defined by

$$
\left\{x_{i}, x_{j}\right\}=\epsilon_{i j k} x_{k},
$$


which allows to express the action of the rotation vector fields $R_{k}$ on the complex scalar field $\phi$ in a Hamiltonian way,

$$
R_{k} \phi=\left\{x_{k}, \phi\right\} .
$$

Bracket (2.19) can be also described more invariantly if we introduce the so-called moment map $M$ which is one of the central notions of the Poisson geometry. The moment map is precisely used in the situations when a Lie algebra $\mathcal{G}$ acts on a Poisson manifold $P$ in a Hamiltonian way. In this case, every element $\chi \in \mathcal{G}$ acts on a function $\phi$ on $P$ via the Hamiltonian vector field $v_{\chi} \phi:=\left\{h_{\chi}, \phi\right\}$ and the moment map $M$ is the book-keeping device of all Hamiltonians $h_{\chi}$. Concretely, it is a map from $P$ to the dual $\mathcal{G}^{*}$ of a Lie algebra $\mathcal{G}$ such that $h_{\chi}=\langle M, \chi\rangle$ for every $\chi \in \mathcal{G}$. In the case when the Poisson manifold is $S^{2}$ acted upon by the Lie algebra $\mathcal{G}=s u(2)$, it is convenient to identify the dual $\mathcal{G}^{*}$ with $\mathcal{G}$ via the Killing form on $s u(2)$ which permits to view $M$ as a traceless idempotent Hermitian $2 \times 2$ matrix the entries of which are the complex functions $x_{3}, x_{ \pm}:=x_{1} \pm \mathrm{i} x_{2}$ on the sphere,

$$
M=\left(\begin{array}{cc}
x_{3} & x_{-} \\
x_{+} & -x_{3}
\end{array}\right) .
$$

We observe that the moment map $M$ indeed "contains" all three Hamiltonians $x_{1}, x_{2}, x_{3}$ of the basic infinitesimal rotations $R_{k}, k=1,2,3$ and it turns out that it "contains" also the Hamiltonians for any linear combination of $R_{k}$ (denoted $V, V_{1}, V_{2}$, etc., in what follows).

The set of defining Poisson brackets (2.19) can be then rewritten in several equivalent ways which are useful in different contexts. For example,

$$
\left\{\operatorname{tr}\left(V_{1} M\right), \operatorname{tr}\left(V_{2} M\right)\right\}=-\operatorname{itr}\left(\left[V_{1}, V_{2}\right] M\right),
$$

where $V_{1}, V_{2}$ are arbitrary traceless Hermitian matrices representing the Lie algebra $s o(3) \equiv s u(2)$ in the spin $\frac{1}{2}$ representation (the choice of the Pauli matrices for $V_{1}, V_{2}$ gives readily (2.19)). Other descriptions of this fundamental Poisson structure on the sphere are matrix-like, e.g.,

$$
\{\operatorname{tr}(V M), M\}=\mathrm{i}[V, M]
$$

or

$$
\{M, M\}=2 \mathrm{i} M,
$$

the last representation should be read in components as

$$
\sum_{j}\left\{M_{i j}, M_{j k}\right\}=2 \mathrm{i} M_{i k}
$$

Finally, it holds true also

$$
\left\{M_{i j}, M_{k l}\right\}=\mathrm{i} \delta_{j k} M_{i l}-\mathrm{i} \delta_{i l} M_{k j} .
$$

In what follows, it will be convenient to represent the electromagnetic potential $A_{k}$ also by a traceless Hermitian matrix $A$,

$$
A \equiv\left(\begin{array}{cc}
A_{3} & A_{1}-\mathrm{i} A_{2} \\
A_{1}+\mathrm{i} A_{2} & -A_{3}
\end{array}\right) .
$$

By using (2.20), scalar field strength (2.13) can be then written as

$$
F(A)=\frac{\mathrm{i}}{2} M_{k l}\left\{M_{l p}, A_{p k}\right\} \equiv-\frac{\mathrm{i}}{2} \operatorname{tr}(M\{M, A\})
$$

and the new form of manifestly $S O(3)$ invariant action (2.12) can be recast as

$$
S(\phi, A):=\frac{1}{4} \int d \mu_{S^{2}}\left(\|\{M, \phi\}-\mathrm{i} A \phi\|^{2}-\frac{1}{2 e^{2}} \operatorname{tr}^{2}(M\{M, A\})\right) .
$$

Here,

$$
\|\{M, \phi\}-\mathrm{i} A \phi\|^{2}:=\operatorname{tr}((\{M, \bar{\phi}\}+\mathrm{i} A \bar{\phi})(\{M, \phi\}-\mathrm{i} A \phi)) .
$$


Consider the following infinitesimal variations of the fields $\phi$ and $A$ :

$$
\delta_{V} \phi:=\{\operatorname{tr}(V M), \phi\}, \quad \delta_{V} A:=-\mathrm{i}[V, A]+\{\operatorname{tr}(V M), A\} .
$$

Here, an arbitrary element $V$ of $s o(3) \equiv \operatorname{Lie}(S O(3))$ is viewed as the traceless Hermitian matrix in the spin $\frac{1}{2}$ representation of $s o(3)$; in particular, the choice of the Pauli matrices $V=\sigma_{k} / 2$ gives

$$
\delta_{k} \phi:=\left\{x_{k}, \phi\right\}=R_{k} \phi, \quad \delta_{k} A_{l}:=\epsilon_{k m l} A_{m}+\left\{x_{k}, A_{l}\right\} .
$$

Notice also that $s o(3)$ variation (2.32) of $A$ is induced from the Lie derivative of the potential 1-form $A_{\mu} d \xi^{\mu}$

$$
\delta_{k}\left(A_{\mu} d \xi^{\mu}\right) \equiv\left(\iota_{R_{k}} d+d \iota_{R_{k}}\right)\left(A_{\mu} d \xi^{\mu}\right) .
$$

We now wish to show that action (2.29) is $s o(3)$ invariant with respect to variations (2.31) of the interacting fields $\phi$ and $A$,

$$
\delta_{V} S \equiv S\left(\phi+\delta_{V} \phi, A+\delta_{V} A\right)-S(\phi, A)=0 .
$$

To do that, we use Eq. (2.23) and we calculate

$$
\{\operatorname{tr}(V M),\{M, \phi\}\}=\mathrm{i}[V,\{M, \phi\}]+\{M,\{\operatorname{tr}(V M), \phi\}\}
$$

and

$$
\begin{gathered}
\{\operatorname{tr}(V M), \operatorname{tr}(M\{M, A\})\}= \\
=\operatorname{tr}(\mathrm{i}[V, M]\{M, A\}+M\{\mathrm{i}[V, M], A\}+M\{M,\{\operatorname{tr}(V M), A\}\})= \\
=\operatorname{tr}(-M\{M, \mathrm{i}[V, A]\}+M\{M,\{\operatorname{tr}(V M), A\}\}) .
\end{gathered}
$$

From (2.35), (2.36), and (2.31), we then derive

$$
\begin{gathered}
\left\{M, \delta_{V} \phi\right\}=\{\operatorname{tr}(V M),\{M, \phi\}\}-\mathrm{i}[V,\{M, \phi\}], \\
\operatorname{tr}\left(M\left\{M, \delta_{V} A\right\}\right)=\{\operatorname{tr}(V M), \operatorname{tr}(M\{M, A\})\} .
\end{gathered}
$$

Relations (2.37) and (2.38) imply

$$
\delta_{V} S=\frac{1}{4} \int d \mu_{S^{2}}\left\{\operatorname{tr}(V M),\|\{M, \phi\}-\mathrm{i} A \phi\|^{2}-\frac{1}{2} \operatorname{tr}^{2}(M\{M, A\})\right\} .
$$

We finish the proof of the fact that $\delta_{V} S=0$ by exploiting the $s o(3)$ invariance of the measure $d \mu_{S^{2}}$,

$$
0=\delta_{V}\left(\int d \mu_{S^{2}} f\right)=\int d \mu_{S^{2}} \delta_{V} f=\int d \mu_{S^{2}}\{\operatorname{tr}(V M), f\} .
$$

Relation (2.40) holds for any function $f$ on $S^{2}$, in particular for that appearing in (2.39).

Now we verify the gauge invariance of the action $S(\phi, A)$ with respect to the following gauge transformation depending on an arbitrary function $\rho$ on $S^{2}$,

$$
A \rightarrow A+\{M, \rho\}, \quad \phi \rightarrow e^{\mathrm{i} \rho} \phi .
$$

The check of the invariance of the matter kinetic term is trivial; however, a little bit more work is needed to establish the invariance of the field strength.

Because the moment map $M$ squares to the unit matrix, we obtain, respectively, for every function $f$ on $S^{2}$ and for every matrix function $T$ on $S^{2}$,

$$
\begin{gathered}
0=\operatorname{tr}\left\{M^{2}, f\right\}=\operatorname{tr}(M\{M, f\}+\{M, f\} M)=2 \operatorname{tr}(M\{M, f\}), \\
0=\operatorname{tr}\left\{M^{2}, T\right\}=\operatorname{tr}(M\{M, T\})-\operatorname{tr}(M\{T, M\}) .
\end{gathered}
$$

Relations (2.42), (2.24), and also (2.43), considered for $T=\{M, f\}$, then imply

$$
2 \operatorname{tr}(M\{M,\{M, f\}\})=\operatorname{tr}(M\{\{M, M\}, f\})=2 \operatorname{itr}(M\{M, f\})=0 .
$$

Obviously, Equation (2.44) guarantees the invariance of field strength (2.28) with respect to gauge transformation (2.41). For that matter, we should perhaps recall that the field $A$ is constrained by the 
constraint $x_{k} A_{k}=0$ which can be rewritten also as

$$
\operatorname{tr}(M A)=0 .
$$

We observe from (2.42) that this constraint is also preserved by gauge transformation (2.41) as it should.

Remark 2. There is an alternative way of writing action (2.29) in terms of the Hodge dual fields $B_{k}$ given by (2.17). Introducing the traceless Hermitian matrix $B$ by

$$
B \equiv\left(\begin{array}{cc}
B_{3} & B_{1}-\mathrm{i} B_{2} \\
B_{1}+\mathrm{i} B_{2} & -B_{3}
\end{array}\right),
$$

relations (2.17) can be rewritten as

$$
B:=\frac{\mathrm{i}}{2}[M, A], \quad A=-\frac{\mathrm{i}}{2}[M, B]
$$

and action (2.29) as

$$
S(\phi, B)=\frac{1}{4} \int d \mu_{S^{2}}\left(\left\|\{M, \phi\}-\frac{1}{2}[M, B] \phi\right\|^{2}+\frac{1}{2 e^{2}} \operatorname{tr}^{2}\{M, B\}\right) .
$$

In this dual way of writing the action of the scalar electrodynamics on the sphere, the kinetic term of the gauge field gets simpler at the price of rendering the matter kinetic term slightly more complicated. This alternative action (2.48) is of course also $s o(3)$ invariant and gauge invariant with respect to the following so(3) and gauge transformations of the fields $\phi$ and $B$,

$$
\begin{gathered}
\delta_{V} \phi:=\{\operatorname{tr}(V M), \phi\}, \quad \delta_{V} B:=-\mathrm{i}[V, B]+\{\operatorname{tr}(V M), B\}, \\
\phi \rightarrow e^{\mathrm{i} \rho} \phi, \quad B \rightarrow B-\frac{\mathrm{i}}{2}[M,\{M, \rho\}] .
\end{gathered}
$$

Notice that these transformations respect the constraint $\operatorname{tr}(M B)=0$.

\section{Scalar electrodynamics on the fuzzy sphere}

Now we are going to present a new construction of a purely bosonic scalar electrodynamics on the fuzzy sphere $S_{N}^{2} \cdot{ }^{15,27}$ Recall that $S_{N}^{2}$ is the noncommutative manifold resulting from the quantization of $S^{2}$ induced by Poisson brackets (2.19). The linear $S O(3)$-equivariant quantization map $Q_{N}$ associates to smooth functions $f$ on $S^{2}$ sequences of $N \times N$-matrices $Q_{N}(f)$ which are called the quantized or fuzzy functions. We shall not need an explicit formula for the quantization map $Q_{N}$ but we do need three basic properties of it,

$$
\begin{gathered}
Q_{N}(f) Q_{N}(g)=Q_{N}(f g)+O\left(\frac{2}{\sqrt{N^{2}-1}}\right), \\
{\left[Q_{N}(f), Q_{N}(g)\right]=\mathrm{i} \frac{2}{\sqrt{N^{2}-1}} Q_{N}(\{f, g\})+O\left(\frac{4}{N^{2}-1}\right),} \\
\frac{1}{\pi} \int_{S^{2}} d \mu_{S^{2}} f=\frac{2}{\sqrt{N^{2}-1}} \operatorname{Tr}\left(Q_{N}(f)\right)+O\left(\frac{4}{N^{2}-1}\right) .
\end{gathered}
$$

Obviously, the parameter $2 / \sqrt{N^{2}-1}$ plays the role of the Planck constant for the quantization map $Q_{N}$. It is also important to stress that Tr stands for the trace of the $N \times N$ matrices while, throughout this paper, we reserve the symbol tr for the trace of $2 \times 2$ matrices.

To give a flavor, what the map $Q_{N}$ is about, let us make explicit the quantized versions of the functions $1, x_{3}, x_{1} \pm \mathrm{i} x_{2}$ on $S^{2}$, 


$$
\begin{gathered}
Q_{N}(1)=\mathbf{1}_{N}, \\
Q_{N}\left(x_{3}\right)_{i j}=\frac{1}{\sqrt{N^{2}-1}}(N+1-2 j) \delta_{i j}, \\
Q_{N}\left(x_{1}+\mathrm{i} x_{2}\right)_{i j}=\frac{2}{\sqrt{N^{2}-1}} \sqrt{(j-1)(N-j+1)} \delta_{i, j-1}, \\
Q_{N}\left(x_{1}-\mathrm{i} x_{2}\right)=Q_{N}\left(x_{1}+\mathrm{i} x_{2}\right)^{\dagger},
\end{gathered}
$$

where $\mathbf{1}_{N}$ stands for the unit $N \times N$-matrix. In particular, it is then easy to verify that it holds the emblematic fuzzy sphere relation

$$
Q_{N}\left(x_{1}\right)^{2}+Q_{N}\left(x_{2}\right)^{2}+Q_{N}\left(x_{3}\right)^{2}=Q_{N}(1) .
$$

In what follows, we shall adopt a notation keeping the dependence on $N$ tacit,

$$
\hat{x}_{k}:=Q_{N}\left(x_{k}\right) .
$$

It can be easily checked that the following Hermitian matrices $L_{k}$

$$
L_{k}:=\frac{\sqrt{N^{2}-1}}{2} \hat{x}_{k},
$$

realize an $\mathrm{N}$-dimensional unitary representation of the Lie algebra $s o(3)$. This fact is compatible with property (2.52) and with definition (2.19).

Let us now construct a field theory on the fuzzy sphere, the fields of which are a complex $N \times N$ matrix $\hat{\phi} \equiv Q_{N}(\phi)$ and three Hermitian $N \times N$ matrices $\hat{A}_{k} \equiv Q_{N}\left(A_{k}\right)$ obeying a constraint

$$
\hat{x}_{k} \hat{A}_{k}+\hat{A}_{k} \hat{x}_{k}+\frac{2}{\sqrt{N^{2}-1}} \hat{A}_{k} \hat{A}_{k}=0 .
$$

For the action functional, we take

$$
S_{N}\left(\hat{\phi}, \hat{A}_{k}\right)=\frac{\pi}{\sqrt{N^{2}-1}} \operatorname{Tr}\left(\left(\left[L_{k}, \hat{\phi}\right]+\hat{A}_{k} \hat{\phi}\right)^{\dagger}\left(\left[L_{k}, \hat{\phi}\right]+\hat{A}_{k} \hat{\phi}\right)-\frac{1}{e^{2}} F(\hat{A})^{2}\right),
$$

where the $N \times N$ matrix $F(\hat{A})$ defined by

$$
F(\hat{A})=-\mathrm{i} \frac{2}{\sqrt{N^{2}-1}} \epsilon_{k l m}\left(\left(L_{k}+\hat{A}_{k}\right)\left(L_{l}+\hat{A}_{l}\right)\left(L_{m}+\hat{A}_{m}\right)-L_{k} L_{l} L_{m}\right)
$$

plays the role of the fuzzy field strength. Fuzzy action (2.62) is invariant with respect to the following $s o(3)$ field variations

$$
\delta_{p} \hat{\phi}:=-\mathrm{i}\left[L_{p}, \hat{\phi}\right], \quad \delta_{p} \hat{A}_{k}=\epsilon_{p l k} \hat{A}_{l}-\mathrm{i}\left[L_{p}, \hat{A}_{k}\right],
$$

because it can be easily checked that

$$
\begin{gathered}
\delta_{p}\left(\left[L_{k}, \hat{\phi}\right]+\hat{A}_{k} \hat{\phi}\right) \equiv\left[L_{k}, \delta_{p} \hat{\phi}\right]+\left(\delta_{p} \hat{A}_{k}\right) \hat{\phi}+\hat{A}_{k} \delta_{p} \hat{\phi}= \\
\left.=\epsilon_{p l k}\left(\left[L_{l}, \hat{\phi}\right]+\hat{A}_{l} \hat{\phi}\right]\right)-\mathrm{i}\left[L_{p},\left[L_{k}, \hat{\phi}\right]+\hat{A}_{k} \hat{\phi}\right]
\end{gathered}
$$

and

$$
\delta_{p} F(\hat{A})=-\mathrm{i}\left[L_{p}, F(\hat{A})\right] .
$$

The $s o(3)$ invariance of constraint (2.61) can be verified in a similar way.

Our fuzzy action (2.62) as well as constraint (2.61) can be easily checked to be also gauge invariant with respect to the following gauge transformation:

$$
\hat{\phi} \rightarrow U \hat{\phi}, \quad \hat{A}_{k} \rightarrow U \hat{A}_{k} U^{-1}-\left[L_{k}, U\right] U^{-1},
$$

where $U$ is an arbitrary unitary $N \times N$ matrix. Notice, in particular, that the noncommutative field strength $F(\hat{A})$ transforms under $(2.67)$ as

$$
F(\hat{A}) \rightarrow U F(\hat{A}) U^{-1} .
$$


There exists a more compact way of writing fuzzy action (2.62) not only in the spirit of commutative action (2.29) but also in the spirit of the matrix model philosophy of Ref. 33. So, as in the commutative case, we define $2 \times 2$ matrices

$$
\hat{A} \equiv\left(\begin{array}{cc}
\hat{A}_{3} & \hat{A}_{1}-\mathrm{i} \hat{A}_{2} \\
\hat{A}_{1}+\mathrm{i} \hat{A}_{2} & -\hat{A}_{3}
\end{array}\right), \quad \hat{M} \equiv\left(\begin{array}{cc}
\hat{x}_{3} & \hat{x}_{1}-\mathrm{i} \hat{x}_{2} \\
\hat{x}_{1}+\mathrm{i} \hat{x}_{2} & -\hat{x}_{3}
\end{array}\right)
$$

and

$$
\hat{E}:=\hat{M}+\frac{2}{\sqrt{N^{2}-1}} \hat{A} .
$$

In terms of the matrix $\hat{E}$, action (2.62) can be expressed compactly as

$$
S_{N}(\hat{\phi}, \hat{E})=\frac{\pi}{2} \frac{\sqrt{N^{2}-1}}{2} \operatorname{Tr}\left(\operatorname{tr}\left((\hat{E} \hat{\phi}-\hat{\phi} \hat{M})^{\dagger}(\hat{E} \hat{\phi}-\hat{\phi} \hat{M})\right)-\frac{N^{2}-1}{16 e^{2}} \operatorname{tr}^{2}\left(\hat{E}^{3}-\hat{M}^{3}\right)\right),
$$

where tr means the trace of $2 \times 2$ matrices and Tr the trace of $N \times N$ matrices.

Our last task in this section is to show that in the limit of large $N$, matrix models (2.62) and (2.71) give scalar electrodynamics (2.29) on the ordinary sphere. The large $N$ limit of the kinetic term is easy to establish since relations (2.51), (2.52), and (2.60) directly give

$$
\left[L_{k}, \hat{\phi}\right]+\hat{A}_{k} \hat{\phi}=Q_{N}\left(\mathrm{i}\left\{x_{k}, \phi\right\}+A_{k} \phi\right)+O\left(\frac{2}{\sqrt{N^{2}-1}}\right) .
$$

A little more work is necessary to find the large $N$ limit of the field strength

$$
F(\hat{A})=Q_{N}\left(\epsilon_{k l m} x_{k}\left\{x_{l}, A_{m}\right\}\right)+O\left(\frac{2}{\sqrt{N^{2}-1}}\right) .
$$

The derivation of (2.73) is not only based on the same relations (2.51), (2.52), and (2.60) as before but also on constraint (2.61) which itself can be written as

$$
0=\hat{x}_{k} \hat{A}_{k}+\hat{A}_{k} \hat{x}_{k}+\frac{2}{\sqrt{N^{2}-1}} \hat{A}_{k} \hat{A}_{k}=2 Q_{N}\left(x_{k} A_{k}\right)+O\left(\frac{2}{\sqrt{N^{2}-1}}\right) .
$$

Finally, in the limit of large $N$, the trace $\operatorname{Tr}$ approaches the integral over the sphere following relation (2.53) and this is the last ingredient needed to establish the correct $N \rightarrow \infty$ limit of fuzzy action (2.62).

\section{FUZZY SUPERSYMMETRIC SCHWINGER MODEL}

\section{A. Supermatrices and the supersphere $S^{2 \mid 2}$}

By an even $(2 \mid 1) \times(2 \mid 1)$ supermatrix over a complex Grassmann algebra, we mean a square matrix $\mathcal{V}$ of the block form

$$
\mathcal{V}=\left(\begin{array}{ll}
A & B \\
C & D
\end{array}\right),
$$

where $A$ is a $2 \times 2$ square matrix with even entries, the column vector $B$ and the row vector $C$ have odd entries, and $D$ is an even element of the Grassmann algebra. In what follows, we shall exclusively consider the Grassmann algebras equipped with the so-called graded involution. The latter was introduced in Ref. 29 and satisfies

$$
\overline{a b}=\bar{a} \bar{b}, \quad \overline{\bar{a}}=(-1)^{\operatorname{deg}(a)} a .
$$

By definition, the Hermitian conjugated matrix $\mathcal{V}^{\dagger}$ has entries which fulfil

$$
\left(\mathcal{V}^{\dagger}\right)_{i j}:=\operatorname{sign}(i-j) \overline{\mathcal{V}}_{j i},
$$


where $\operatorname{sign}(i-j)$ takes value 1 if $i \geq j$ and -1 otherwise. Every Hermitian $(2 \mid 1) \times(2 \mid 1)$ supermatrix $\mathcal{V}^{\dagger}=\mathcal{V}$ can be unambiguously represented as a sum

$$
\mathcal{V}=\operatorname{str}(\mathcal{V}) \mathbf{1}+\mathcal{V}_{v}+\mathcal{V}_{s},
$$

where the supertrace is defined by

$$
\operatorname{str}(\mathcal{V}):=\operatorname{tr}(A)-D
$$

and $\mathcal{V}_{v}, \mathcal{V}_{s}$ are traceless Hermitian supermatrices of the respective forms

$$
\mathcal{V}_{v}=\left(\begin{array}{ccc}
v_{3} & \bar{v} & -\bar{v} \\
v & -v_{3} & v \\
v & \bar{v} & 0
\end{array}\right), \quad \mathcal{V}_{s}=\left(\begin{array}{ccc}
v_{0} & 0 & \vartheta \\
0 & v_{0} & -\bar{\vartheta} \\
\bar{\vartheta} & \vartheta & 2 v_{0}
\end{array}\right) .
$$

Now it can be easily checked that the ordinary commutator of two even traceless Hermitian supermatrices of the $v$-type ${ }^{40}$ is again an even traceless Hermitian supermatrix of the $v$-type. The even traceless Hermitian supermatrices of the $v$-type thus form a Lie superalgebra referred to as $\operatorname{uosp}(2 \mid 1)$. It can be also verified that the commutator of the matrix of the $v$-type with the matrix of the $s$-type is of the $s$-type; therefore, the space of the matrices of the $s$-type is the representation of $\operatorname{uosp}(2 \mid 1)$ called the superspinorial representation.

The supersphere $S^{2 \mid 2}$ is a supermanifold generated by three even real variables $y_{k}, k=1,2,3$ and a pair of graded complex conjugated odd variables $\theta, \bar{\theta}$ fulfilling one constraint

$$
y_{1}^{2}+y_{2}^{2}+y_{3}^{2}+2 \theta \bar{\theta}=1 .
$$

It will prove extremely useful to organize the generators $y_{k}, \theta, \bar{\theta}$ into the even traceless Hermitian supermatrix of the $v$-type as follows:

$$
\mathcal{M}=\left(\begin{array}{ccc}
y_{3} & y_{1}-\mathrm{i} y_{2} & -\bar{\theta} \\
y_{1}+\mathrm{i} y_{2} & -y_{3} & \theta \\
\theta & \bar{\theta} & 0
\end{array}\right) .
$$

The $\operatorname{uosp}(2 \mid 1)$ Kirillov-Kostant-Souriau Poisson structure on $S^{2 \mid 2}$ is then defined by the following bracket:

$$
\left\{\operatorname{str}\left(\mathcal{V}_{1} \mathcal{M}\right), \operatorname{str}\left(\mathcal{V}_{2} \mathcal{M}\right)\right\}:=-\operatorname{istr}\left(\left[\mathcal{V}_{1}, \mathcal{V}_{2}\right] \mathcal{M}\right), \quad \mathcal{V}_{1}, \mathcal{V}_{2} \in \text { uosp }(2 \mid 1)
$$

Thus, the Hamiltonian generating the action of $\mathcal{V}$ on the supersphere $S^{2 \mid 2}$ via the Poisson bracket is $\operatorname{str}(\mathcal{V} \mathcal{M})$ and hence, $\mathcal{M} \in \operatorname{uosp}(2 \mid 1)$ is nothing but the moment map of this action. Other description of this fundamental Poisson structure on the supersphere is matrix-like, e.g.,

$$
\{\operatorname{str}(\mathcal{V} \mathcal{M}), \mathcal{M}\}=\mathrm{i}[\mathcal{V}, \mathcal{M}]
$$

or

$$
\{\mathcal{M}, \mathcal{M}\}=\frac{3}{2} \mathrm{i} \mathcal{M} .
$$

Although we do not need it, we list for completeness the Poisson brackets of the generators $y_{k}, \theta, \bar{\theta}$ as they follow from general formula (3.9),

$$
\begin{gathered}
\left\{y_{k}, y_{l}\right\}=\epsilon_{k l m} y_{m}, \quad\left\{y_{3}, \theta\right\}=-\frac{\mathrm{i}}{2} \theta, \quad\left\{y_{3}, \bar{\theta}\right\}=+\frac{\mathrm{i}}{2} \bar{\theta}, \\
\left\{y_{1}+\mathrm{i} y_{2}, \bar{\theta}\right\}=-\mathrm{i} \theta, \quad\left\{y_{1}-\mathrm{i} y_{2}, \theta\right\}=-\mathrm{i} \bar{\theta}, \quad\left\{y_{1}+\mathrm{i} y_{2}, \theta\right\}=0=\left\{y_{1}-\mathrm{i} y_{2}, \bar{\theta}\right\}, \\
\{\theta, \theta\}=-\frac{\mathrm{i}}{2}\left(y_{1}+\mathrm{i} y_{2}\right), \quad\{\bar{\theta}, \bar{\theta}\}=+\frac{\mathrm{i}}{2}\left(y_{1}-\mathrm{i} y_{2}\right), \quad\{\theta, \bar{\theta}\}=\frac{\mathrm{i}}{2} y_{3} .
\end{gathered}
$$

Unlike the $\operatorname{so}(3)$ moment map $M$ considered in Sec. II, the $\operatorname{uosp}(2 \mid 1)$ moment map $\mathcal{M}$ given by (3.8) is not idempotent. In fact, the square of $\mathcal{M}$ is nontrivial and plays a very important role in the construction of the supersymmetric invariants. Thus, we define

$$
\mathcal{K}:=\mathcal{M}^{2}-2
$$


and we find that $\mathcal{K}$ is the Hermitian supermatrix of the $s$-type. For completeness, we describe it explicitly

$$
\mathcal{K}=\left(\begin{array}{ccc}
w & 0 & \zeta \\
0 & w & -\bar{\zeta} \\
\bar{\zeta} & \zeta & 2 w
\end{array}\right)
$$

where

$$
\zeta:=\theta\left(y_{1}-\mathrm{i} y_{2}\right)-\bar{\theta} y_{3}, \quad \bar{\zeta}:=\theta y_{3}+\bar{\theta}\left(y_{1}+\mathrm{i} y_{2}\right), \quad w=-1-\theta \bar{\theta} .
$$

We finish this section by listing useful formulae expressing the Poisson brackets involving the matrix $\mathcal{K}$,

$$
\{\mathcal{K}, \mathcal{K}\}=-\frac{\mathrm{i}}{2} \mathcal{M}, \quad\{\mathcal{K}, \mathcal{M}\}=\{\mathcal{M}, \mathcal{K}\}=\frac{\mathrm{i}}{2} \mathcal{K} .
$$

\section{B. Supersymmetric electrodynamics on $S^{2 \mid 2}$}

By using the supermatrix $\mathcal{K}$ defined by (3.13) and (3.14), a manifestly $u o s p(2 \mid 1)$ invariant action of a free massless complex scalar superfield $\Phi$ on the supersphere was written in Ref. 26. It reads

$$
S_{0}(\Phi)=\operatorname{str} \int d \mu_{S^{2 \mid 2}}\{\mathcal{K}, \bar{\Phi}\}\{\mathcal{K}, \Phi\}
$$

where the $\operatorname{uosp}(2 \mid 1)$ invariant measure on the supersphere $S^{2 \mid 2}$ is defined by ${ }^{14,26}$

$$
d \mu_{S^{2 \mid 2}}:=d y_{1} d y_{2} d y_{3} d \theta d \bar{\theta} \delta\left(y_{1}^{2}+y_{2}^{2}+y_{3}^{2}+2 \theta \bar{\theta}-1\right) .
$$

The immediate consequence of the invariance of the measure $d \mu_{S^{2 \mid 2}}$ is the formula

$$
\int d \mu_{S^{2 \mid 2}}\{\mathcal{M}, f\}=0
$$

valid for all functions $f$ on the supersphere (cf. (2.40)). Thanks to (3.19) and to the following identity:

$$
\{\mathcal{K},\{\operatorname{str}(\mathcal{V} \mathcal{M}), \Phi\}\}=\{\operatorname{str}(\mathcal{V} \mathcal{M}),\{\mathcal{K}, \Phi\}\}-\mathrm{i}[\mathcal{V},\{\mathcal{K}, \Phi\}]
$$

derived from (3.10) and from (3.13), free action (3.17) can be easily checked to be invariant with respect to the $\operatorname{uos} p(2 \mid 1)$ action on $\Phi$ defined by

$$
\delta_{\mathcal{V}} \Phi:=\{\operatorname{str}(\mathcal{V} \mathcal{M}), \Phi\}
$$

Let us gauge the global $U(1)$ symmetry $\Phi \rightarrow \exp \varrho_{0} \Phi$ of action (3.17) by introducing a multiplet of odd and even gauge superfields $C, \bar{C}, C_{0}$ arranged in the matrix of the $s$-type,

$$
C:=\left(\begin{array}{ccc}
C_{0} & 0 & C \\
0 & C_{0} & -\bar{C} \\
\bar{C} & C & 2 C_{0}
\end{array}\right) .
$$

We require, moreover, that $C$ is constrained by

$$
\operatorname{str}(\mathcal{K} C)=0 .
$$

Consider now the following action in which the $s$-type matrix superfield $C$ is viewed as external:

$$
S_{\text {ext }}(\Phi, C)=\operatorname{str} \int d \mu_{S^{2 \mid 2}}(\{\mathcal{K}, \bar{\Phi}\}+\mathrm{i} C \bar{\Phi})(\{\mathcal{K}, \Phi\}-\mathrm{i} C \Phi) .
$$

It is easy to check the symmetry of the action $S_{\text {ext }}$ with respect to the following gauge transformations:

$$
\Phi \rightarrow \varepsilon^{\mathrm{i} \varrho} \Phi, \quad C \rightarrow C+\{\mathcal{K}, \varrho\}
$$

where $\varrho$ is an arbitrary even real function on $S^{2 \mid 2}$. 
Now we are going to concentrate to the problem how to render the supermatrix gauge superfield $C$ dynamical. Said in other words, we must construct a viable manifestly supersymmetric kinetic term for the gauge superfield $C$ to be added to supersymmetric action (3.24). One way of solving this problem was shown in Ref. 25, the other, and drastically simpler, is presented in this paper. Why we have not seen the simpler solution while writing the former paper? ${ }^{25}$ Well, we were not aware at that time of a possibility to use Poisson geometry as a very efficient conceptual and technical tool for constructing superinvariant Lagrangians. We have first used this tool only recently in the context of supersymmetric $\sigma$-model ${ }^{26}$ and the present article constitutes another proof of its efficiency. Thus, we introduce here a concept of a scalar superfield strength $\mathcal{F}(C)$ defined as

$$
\mathcal{F}(C):=\frac{1}{2} \operatorname{str}(\mathcal{M}\{\mathcal{K}, C\}+\{C, \mathcal{K}\} \mathcal{M}) .
$$

For completeness, we detail here this formula in terms of the constituent superfields $C_{0}, C, \bar{C}$ of $C$,

$$
\begin{gathered}
\mathcal{F}(C)=\bar{\theta}\left(\left\{\bar{\zeta}, C_{0}\right\}-\{w, \bar{C}\}\right)+\theta\left(\{w, C\}-\left\{\zeta, C_{0}\right\}\right)+ \\
+y_{3}(\{\zeta, \bar{C}\}+\{\bar{\zeta}, C\})+\left(y_{1}+\mathrm{i} y_{2}\right)\{\zeta, C\}-\left(y_{1}-\mathrm{i} y_{2}\right)\{\bar{\zeta}, \bar{C}\} .
\end{gathered}
$$

This scalar superfield strength $\mathcal{F}(C)$ is really scalar which means that its variation $\delta_{\mathcal{V}} \mathcal{F}$ induced by the vector-like transformation

$$
\delta_{\mathcal{V}} C:=-\mathrm{i}[\mathcal{V}, C]+\{\operatorname{str}(\mathcal{V} \mathcal{M}), C\}
$$

is simply

$$
\delta_{\mathcal{V}} \mathcal{F}(C)=\{\operatorname{str}(\mathcal{V M}), \mathcal{F}(C)\} .
$$

Let us prove formula (3.29) to give an illustration of the efficiency of our compact notation using the supermatrices $\mathcal{M}$ and $\mathcal{K}$. First of all, since $\mathcal{K}=\mathcal{M}^{2}-2$, we infer from (3.10) that

$$
\{\operatorname{str}(\mathcal{V} \mathcal{M}), \mathcal{K}\}=i[\mathcal{V}, \mathcal{K}] .
$$

Then, we find from (3.10) and (3.30),

$$
\begin{gathered}
\{\operatorname{str}(\mathcal{V} \mathcal{M}), \operatorname{str}(\mathcal{M}\{\mathcal{K}, C\}\})=\operatorname{str}(\mathrm{i}[\mathcal{V}, \mathcal{M}]\{\mathcal{K}, C\})+\operatorname{str}(\mathcal{M}\{\mathrm{i}[\mathcal{V}, \mathcal{K}], C\})+ \\
+\operatorname{str}(\mathcal{M}\{\mathcal{K},\{\operatorname{str}(\mathcal{V} \mathcal{M}), C\}\})=\operatorname{str}(\mathrm{i}[\mathcal{V}, \mathcal{M}\{\mathcal{K}, C\}])-\operatorname{str}(\mathcal{M}\{\mathcal{K}, \mathrm{i}[\mathcal{V}, C]\})+ \\
+\operatorname{str}(\mathcal{M},\{\mathcal{K},\{\operatorname{str}(\mathcal{V} \mathcal{M}), C\}\})=\operatorname{str}\left(\mathcal{M}\left\{\mathcal{K}, \delta_{\mathcal{V}} C\right\}\right)
\end{gathered}
$$

Much in the same way, we find

$$
\{\operatorname{str}(\mathcal{V} \mathcal{M}), \operatorname{str}(\{C, \mathcal{K}\} \mathcal{M})\}=\operatorname{str}\left(\left\{\delta_{\mathcal{V}} C, \mathcal{K}\right\} \mathcal{M}\right) .
$$

Eqs. (3.31) and (3.32) then clearly imply (3.29).

Let us now prove the invariance of the scalar superfield strength $\mathcal{F}(C)$ with respect to gauge transformation (3.25). By using the Jacobi identity in two alternative forms

$$
\begin{aligned}
& \{a,\{b, \rho\}\}=\{\{a, b\}, \rho\}+\{\{\rho, a\}, b\}, \\
& \{\{\rho, b\}, a\}=\{\rho,\{b, a\}\}+\{b,\{a, \rho\}\},
\end{aligned}
$$

valid for arbitrary functions $a, b, \rho$ on $S^{2 \mid 2}$ with $\rho$ even, we infer from (3.16)

$$
\operatorname{str}(\mathcal{M}\{\mathcal{K},\{\mathcal{K}, \rho\}\}-\{\{\rho, \mathcal{K}\}, \mathcal{K}\} \mathcal{M})=\operatorname{str}(\mathcal{M}\{\{\mathcal{K}, \mathcal{K}\}, \rho\})=-\frac{\mathrm{i}}{2} \operatorname{str}(\mathcal{M}\{\mathcal{M}, \rho\}) .
$$

Because the supermatrix $\mathcal{M}^{2}$ has constant supertrace (cf., (3.13) and (3.14)), it holds

$$
\operatorname{str}(\mathcal{M}\{\mathcal{M}, \rho\})=\frac{1}{2} \operatorname{str}\left\{\mathcal{M}^{2}, \rho\right\}=0 .
$$

Inserting (3.36) into (3.35), we obtain the gauge invariance of $\mathcal{F}(C)$, hence also the gauge invariance of the following action:

$$
S(\Phi, C)=\operatorname{str} \int d \mu_{S^{212}}\left((\{\mathcal{K}, \bar{\Phi}\}+\mathrm{i} C \bar{\Phi})(\{\mathcal{K}, \Phi\}-\mathrm{i} C \Phi)-\frac{1}{e^{2}}\{\mathcal{K}, \mathcal{F}(C)\}^{2}\right) .
$$

Relations (3.20) and (3.29) then easily imply also the $u \operatorname{sp}(2 \mid 1)$ superinvariance of (3.37). 
Compact and elegant expression (3.37) for the action of the $u o s p(2 \mid 1)$ supersymmetric Schwinger model on the supersphere constitutes the first main result of this article.

Remark 3. The supersymmetric Schwinger model on the supersphere can be rewritten in terms of a dual parametrization of the gauge superfield $C$ much in the spirit of Remark 1 . Thus, define a new $s$-type traceless Hermitian matrix superfield by

$$
\mathcal{E}:=-\mathrm{i}[\mathcal{M}, C] .
$$

Because of constraint (3.23), it holds also

$$
\mathcal{C}:=\mathrm{i}[\mathcal{M}, \mathcal{E}]
$$

and

$$
\operatorname{str}(\mathcal{K} \mathcal{E})=0 .
$$

The duality $C \leftrightarrow \mathcal{E}$ may be called the Hodge one by analogy with the purely bosonic case, albeit we are not aware of its possible interpretation in the language of differential forms. A recent paper ${ }^{5}$ may possibly shed more light on this issue. Finally, the scalar superfield strength $\mathcal{F}$ in terms of $\mathcal{E}$ reads simply

$$
\mathcal{F}(\mathcal{E})=-\frac{\mathrm{i}}{2} \operatorname{str}\{\mathcal{K}, \mathcal{E}\}
$$

and the supersymmetric Schwinger model action becomes

$S(\Phi, \mathcal{E})=\operatorname{str} \int d \mu_{S^{2 \mid 2}}\left((\{\mathcal{K}, \bar{\Phi}\}-[\mathcal{M}, \mathcal{E}] \bar{\Phi})(\{\mathcal{K}, \Phi\}+[\mathcal{M}, \mathcal{E}] \Phi)-\frac{1}{4 e^{2}}\{\mathcal{K}, \operatorname{str}\{\mathcal{K}, \mathcal{E}\}\}^{2}\right)$.

It is in this form that we have presented the manifestly $\operatorname{uosp}(2 \mid 1)$ supersymmetric action of the Schwinger model on $S^{2 \mid 2}$ in the Introduction.

\section{Component expansions}

In this section, we shall work out the action of the supersymmetric electrodynamics on the supersphere in components. We do it starting from dual formulation (3.42) in which the scalar supersymmetric field strength has simpler form. Recall that the gauge field $\mathcal{E}$ is the Hermitian supermatrix of the type $s$,

$$
\mathcal{E}=\left(\begin{array}{ccc}
E_{0} & 0 & E \\
0 & E_{0} & -\bar{E} \\
\bar{E} & E & 2 E_{0}
\end{array}\right),
$$

which verifies the constraint

$$
\operatorname{str}(\mathcal{K} \mathcal{E})=0 .
$$

Constraint (3.44) allows to express the even superfield $E_{0}$ in terms of the (mutually graded conjugated) superfields $E, \bar{E}$ as follows:

$$
E_{0}=\bar{\zeta} E-\zeta \bar{E} .
$$

Scalar superfield strength (3.41) in terms of the constituent superfields $E_{0}, E$, and $\bar{E}$ becomes

$$
\mathcal{F}(\mathcal{E})=\mathrm{i}\left\{w, E_{0}\right\}-\mathrm{i}\{\zeta, \bar{E}\}+\mathrm{i}\{\bar{\zeta}, E\},
$$

or, by using the formula (3.45), as

$$
\mathcal{F}(\mathcal{E})=\frac{1}{2}(\theta E-\bar{\theta} \bar{E})+\mathrm{i}(1+\theta \bar{\theta})(\{\bar{\zeta}, E\}-\{\zeta, \bar{E}\}) .
$$

Here, $\theta, \bar{\theta}$ are related to $\zeta, \bar{\zeta}$ as in (3.15). 
For the component expansions of the superfields $E, \bar{E}$ compatible with the graded conjugation we, choose an Ansätz

$$
\begin{gathered}
E=\mathrm{i} \zeta \tilde{A}_{3}-\mathrm{i} \bar{\zeta}\left(\tilde{A}_{1}-\mathrm{i} \tilde{A}_{2}\right)+2\left(\bar{\xi}\left(y_{1}-\mathrm{i} y_{2}\right)+\xi y_{3}\right) \zeta \bar{\zeta}, \\
\bar{E}=-\mathrm{i} \bar{\zeta} \tilde{A}_{3}-\mathrm{i} \zeta\left(\tilde{A}_{1}+\mathrm{i} \tilde{A}_{2}\right)+2\left(\bar{\xi} y_{3}-\xi\left(y_{1}+\mathrm{i} y_{2}\right)\right) \zeta \bar{\zeta},
\end{gathered}
$$

where the real even component fields $\tilde{A}_{k}$ as well as the mutually conjugated odd colleagues $\xi$ and $\bar{\xi}$ depend just on the variables $y_{k}$. Obviously, component Ansätz (3.48) and (3.49) are not the most general one, since, e.g., the zero order terms in $\zeta, \bar{\zeta}$ expansion are missing but all missing terms can be restored by a gauge transformation $\mathcal{E} \rightarrow \mathcal{E}-\mathrm{i}[\mathcal{M},\{\mathcal{K}, \Lambda\}]$ for an appropriate choice of the even superfunction $\Lambda$. Thus, Ansätz (3.48) and (3.49) are nothing but a variant of the Wess-Zumino gauge.

The evaluation of full scalar superfield strength $\mathcal{F}(\mathcal{E})$ (3.47) for Ansätz (3.48) and (3.49) gives

$$
\mathcal{F}(\mathcal{E})=\mathrm{i} y_{k} \tilde{A}_{k}-\xi \bar{\zeta}-\bar{\xi} \zeta+\mathrm{i}\left(F(\tilde{A})-y_{k} \tilde{A}_{k}+y_{l} \partial_{l}\left(y_{k} \tilde{A}_{k}\right)\right) \zeta \bar{\zeta}
$$

where $F(\tilde{A})$ is nothing but purely bosonic scalar curvature (2.13),

$$
F(\tilde{A}):=\epsilon_{k l m} y_{k}\left\{y_{l}, \tilde{A}_{m}\right\} .
$$

Knowing (3.50), we can now easily complete the evaluation of the component expansion of the gauge kinetic term in action (3.42). It reads

$$
\begin{gathered}
\operatorname{str} \int d \mu_{S^{2 \mid 2}}\{\mathcal{K}, \mathcal{F}(\mathcal{E})\}^{2}=-2 \int d \mu_{S^{2 \mid 2}}\left(2\{\bar{\zeta}, \mathcal{F}(\mathcal{E})\}\{\zeta, \mathcal{F}(\mathcal{E})\}+\{w, \mathcal{F}(\mathcal{E})\}^{2}\right)= \\
\left.=-4 \int d \mu_{S^{2 \mid 2}}(1+\zeta \bar{\zeta})\{\bar{\zeta}, \mathcal{F}(\mathcal{E})\}\{\zeta, \mathcal{F}(\mathcal{E})\}\right)= \\
=-\int d \mu_{S^{2}}\left((F(A)+\rho)^{2}+\left\{y_{k}, \rho\right\}\left\{y_{k}, \rho\right\}+\mathrm{i} \Xi^{\dagger} \sigma_{k}\left\{y_{k}, \Xi\right\}+\Xi^{\dagger} \Xi\right) .
\end{gathered}
$$

Here, $\sigma_{k}$ are the Pauli matrices and $\Xi, \Xi^{\dagger}, \rho$, and $A_{k}$ are defined as

$$
\begin{aligned}
& \Xi:=\left(\begin{array}{l}
\xi \\
\bar{\xi}
\end{array}\right), \quad \Xi^{\dagger}=\left(\begin{array}{ll}
\bar{\xi} & -\xi
\end{array}\right), \\
& \rho:=y_{k} \tilde{A}_{k}, \quad A_{k}:=\tilde{A}_{k}-y_{k} \rho .
\end{aligned}
$$

Note that $\rho$ and $A_{k}$ are, respectively, the radial and the tangential parts of the field $\tilde{A}_{k}$ and $F(A)$ stands for the scalar curvature of the tangential part.

We now proceed to the component expansion of the matter kinetic term. By using (3.8), (3.14), (3.43), and (3.44), we find successively

$$
\begin{gathered}
\operatorname{str} \int d \mu_{S^{212}}((\{\mathcal{K}, \bar{\Phi}\}-[\mathcal{M}, \mathcal{E}] \bar{\Phi})(\{\mathcal{K}, \Phi\}+[\mathcal{M}, \mathcal{E}] \Phi))= \\
=-2 \int d \mu_{S^{212}}((\{\bar{\zeta}, \bar{\Phi}\}+\mathrm{i} \bar{C} \bar{\Phi})(\{\zeta, \Phi\}-\mathrm{i} C \Phi)-(\{\zeta, \bar{\Phi}\}+\mathrm{i} C \bar{\Phi})(\{\bar{\zeta}, \Phi\}-\mathrm{i} \bar{C} \Phi)+ \\
\left.+\left(\{w, \bar{\Phi}\}+\mathrm{i} C_{0} \bar{\Phi}\right)\left(\{w, \Phi\}-\mathrm{i} C_{0} \Phi\right)\right)=-2 \int d \mu_{S^{2 \mid 2}}(1+\theta \bar{\theta}) \times \\
\times((\{\bar{\zeta}, \bar{\Phi}\}+\mathrm{i} \bar{C} \bar{\Phi})(\{\zeta, \Phi\}-\mathrm{i} C \Phi)-(\{\zeta, \bar{\Phi}\}+\mathrm{i} C \bar{\Phi})(\{\bar{\zeta}, \Phi\}-\mathrm{i} \bar{C} \Phi)) .
\end{gathered}
$$

Here, $C=\mathrm{i}[\mathcal{M}, \mathcal{E}]$, or, in detail,

$$
\begin{gathered}
C_{0}=-\mathrm{i} \bar{\theta} \bar{E}+\mathrm{i} \theta E, \\
\bar{C}=-\mathrm{i}(1+\theta \bar{\theta})\left(y_{3} \bar{E}+\left(y_{1}+\mathrm{i} y_{2}\right) E\right), \quad C=\mathrm{i}(1+\theta \bar{\theta})\left(y_{3} E-\left(y_{1}-\mathrm{i} y_{2}\right) \bar{E}\right) .
\end{gathered}
$$

For the component expansion of the complex matter superfield $\Phi$, we choose the following Ansatz (cf. Ref. 14):

$$
\begin{aligned}
& \Phi=\phi+\bar{\theta} \psi_{+}+\theta \psi_{-}+\left(F+y_{k} \partial_{k} \phi\right) \theta \bar{\theta}, \\
& \bar{\Phi}=\bar{\phi}+\bar{\theta} \bar{\psi}_{-}-\theta \bar{\psi}_{+}+\left(\bar{F}+y_{k} \partial_{k} \bar{\phi}\right) \theta \bar{\theta} .
\end{aligned}
$$


The component expansion of the superfields $C, \bar{C}$ is obtained easily from expansions (3.48) and (3.49) of $E, \bar{E}$ and from (3.56),

$$
\begin{aligned}
& \bar{C}=\theta A_{3}+\bar{\theta}\left(A_{1}+\mathrm{i} A_{2}\right)-\bar{\zeta} \rho-2 \mathrm{i} \theta \bar{\theta} \bar{\xi}, \\
& C=\theta\left(A_{1}-\mathrm{i} A_{2}\right)-\bar{\theta} A_{3}-\zeta \rho+2 \mathrm{i} \theta \bar{\theta} \xi .
\end{aligned}
$$

We finally insert (3.57)-(3.60) into (3.55) and find the component expansion of $\operatorname{uosp}(2 \mid 1)$ supersymmetric electrodynamics (3.37),

$$
\begin{aligned}
& S(\phi, \Psi, \tilde{A}, \Xi)=-\mathrm{i} \int d \mu_{S^{2}} \Psi^{\dagger}((\{M, \Psi\}-2 \mathrm{i} A \Psi+2 \mathrm{i} M \rho \Psi)-\mathrm{i} \Psi)+ \\
& +\int d \mu_{S^{2}}\|\{M, \phi\}-2 \mathrm{i} A \phi+2 \mathrm{i} M \rho \phi\|^{2}+2 \mathrm{i} \int d \mu_{S^{2}}\left(\bar{\phi} \Xi^{\dagger} \Psi-\phi \Psi^{\dagger} \Xi\right)+ \\
& +\frac{1}{e^{2}} \int d \mu_{S^{2}}\left((F(A)+\rho)^{2}+\left\{y_{k}, \rho\right\}\left\{y_{k}, \rho\right\}+\mathrm{i} \Xi^{\dagger} \sigma_{k}\left\{y_{k}, \Xi\right\}+\Xi^{\dagger} \Xi\right) .
\end{aligned}
$$

Here,

$$
M=\left(\begin{array}{cc}
y_{3} & y_{1}-\mathrm{i} y_{2} \\
y_{1}+\mathrm{i} y_{2} & -y_{3}
\end{array}\right), \quad A \equiv\left(\begin{array}{cc}
A_{3} & A_{1}-\mathrm{i} A_{2} \\
A_{1}+\mathrm{i} A_{2} & -A_{3}
\end{array}\right),
$$

and $\Psi, \Psi^{\dagger}$ are defined by

$$
\Psi:=\left(\begin{array}{l}
\psi_{-} \\
\psi_{+}
\end{array}\right), \quad \Psi^{\dagger}=\left(\begin{array}{ll}
\bar{\psi}_{-} & \bar{\psi}_{+}
\end{array}\right) .
$$

Up to a simple renormalisation of the coupling constant, expression (3.61) contains at the same time purely bosonic scalar electrodynamics (2.3) as well as the fermionic electrodynamics (the Schwinger model) in the manifestly $s o(3)$ invariant formulation. ${ }^{21}$ We note that the emergence of the Yukawa-like terms $\phi \Psi^{\dagger} \Xi$ is not specifically inherent to the choice of the compact Euclidean "space-time" $S^{2}$ but it appears also in the flat space version of the supersymmetric Schwinger model. ${ }^{9}$

Remark 4. We stress that component action (3.61) is written in the manifestly so(3) invariant formulation ${ }^{21}$ and not in the way as in Eq. (2.1) that can be universally used in every curved background. We have explained in detail the relation between these two formulations in the bosonic case in Section II A of the present paper with the result that formalism (2.1) applied to the round metric $g_{\mu \nu}$ on the sphere yields manifestly $s o(3)$ invariant action (2.12) with manifestly so(3) invariant constraint (2.11). In the presence of fermions, the actions formulated in the universal way for every curved background are technically quite involved and we do not expose them since in the round $S^{2}$ case, they anyway boil down to (3.61). However, the reader may find all details of the construction of the manifestly $s o(3)$ invariant formulation from the universal one in the presence of fermions in Sections 1 and 2 of Ref. 21 The crucial step of the construction is the rewriting of the Dirac operator in a curved background $D=\gamma^{\mu}\left(\partial_{\mu}+\frac{1}{4} \gamma^{\nu} \gamma_{\mu ; \nu}\right)$ as $D=\sigma_{k} R_{k}+1$ for the case of the round metric on $S^{2}$.

In our older paper, ${ }^{25}$ we have constructed a different version of the $\operatorname{uosp}(2 \mid 1)$ supersymmetric electrodynamics on the supersphere than that resumed by actions (3.37) or (3.61). The difference in the final component actions is not that big, as we are going to make explicit soon; nevertheless, from the conceptual point of view the older construction is very different (and much more complicated) than the new one. All difference resides in the gauge field kinetic term: in the new version of the theory, it is given by Equation (3.52) and in the older version, ${ }^{25}$ it has also the structure $\operatorname{str} \int d \mu_{S^{2} \mid 2} T(C)^{2}$, where $T(C)$ is again the Hermitian supermatrix of the $s$-type, but $T(C)$ is not equal to $\{\mathcal{K}, \mathcal{F}(C)\}$ as in (3.37). Instead, it is given by Eq. (67) of Ref. 25 which can be rewritten in our Poisson language as

$T(C)=C+\mathrm{i}\{\mathcal{M}, C\}+\mathrm{i}\{C, \mathcal{M}\}-2\{\mathcal{K},\{\mathcal{K}, C\}+\{C, \mathcal{K}\}\}-2\{\{\mathcal{K}, C\}+\{C, \mathcal{K}\}, \mathcal{K}\}$. 
The reader may check with the help of Eqs. (3.16) that $T(C)$ given by (3.64) is invariant with respect to the gauge transformation $C \rightarrow C+\{\mathcal{K}, \varrho\}$, where $\varrho$ is an arbitrary even real function on $S^{2 \mid 2}$. Moreover, since the $\operatorname{cosp}(2 \mid 1)$-covariance of $T(C)$ is also evident, the expression str $\int d \mu_{S^{2 \mid 2}} T(C)^{2}$ has all properties required for an alternative gauge kinetic term. The component expansion based on Ansatz (3.56) then gives

$$
\begin{gathered}
\frac{1}{36} \operatorname{str} \int d \mu_{S^{2 \mid 2}} T(C)^{2}= \\
=\int d \mu_{S^{2}}\left(F(A)^{2}+\frac{2}{9} \rho F(A)+\frac{1}{9} \rho^{2}+\left\{y_{k}, \rho\right\}\left\{y_{k}, \rho\right\}+\mathrm{i} \Xi^{\dagger} \sigma_{k}\left\{y_{k}, \Xi\right\}+\frac{1}{9} \Xi^{\dagger} \Xi\right) .
\end{gathered}
$$

By comparing new gauge kinetic term (3.52) with old one (3.65), we observe that they coincide up to normalization of certain terms. This circumstance is extremely favorable because by taking a suitable linear combination of the old and the new kinetic terms, we can render all gauge fields massless as in the decompactification limit. ${ }^{9}$ Explicitly, we have

$$
\begin{gathered}
\operatorname{str} \int d \mu_{S^{2 \mid 2}}\left(T(C)^{2}+4\{\mathcal{K}, \mathcal{F}(C)\}^{2}\right)= \\
=32 \int d \mu_{S^{2}}\left(F(A)^{2}+\left\{y_{k}, \rho\right\}\left\{y_{k}, \rho\right\}+\mathrm{i} \Xi^{\dagger} \sigma_{k}\left\{y_{k}, \Xi\right\}\right) .
\end{gathered}
$$

Remark 5. We note that by taking the appropriate linear combination of the old and the new gauge kinetic terms, we did not get rid of the scalar field $\rho$ which is necessary for the $\operatorname{uos} p(2 \mid 1)$ supersymmetry but we did get rid of the unusual coupling of $\rho$ to the gauge field strength $F(A)$ while keeping its standard coupling to the matter fields.

\section{Fuzzy supersphere}

Now we turn to the construction of the supersymmetric electrodynamics on the fuzzy supersphere $S_{N}^{2 \mid 2}$. This task was successfully performed in Ref. 25 for "old" gauge kinetic term (3.65), so here we shall concentrate solely to the fuzzification of "new" kinetic term (3.52). To begin, recall that $S_{N}^{2 \mid 2}$ is the noncommutative supermanifold resulting from the quantization of $S^{2 \mid 2}$ induced by Poisson brackets (3.9) or (3.12). A linear uosp(2|1)-equivariant quantization map $Q_{N}$ associates to smooth superfunctions $f$ on $S^{2 \mid 2}$ sequences of $(N+1 \mid N) \times(N+1 \mid N)$ supermatrices $Q_{N}(f)$ which are called the fuzzy superfunctions. We shall not need an explicit formula for the quantization map $Q_{N}$ but we need three basic properties of it,

$$
\begin{gathered}
Q_{N}(f) Q_{N}(g)=Q_{N}(f g)+O\left(\frac{2}{\sqrt{N^{2}+N}}\right), \\
{\left[Q_{N}(f), Q_{N}(g)\right]=\mathrm{i} \frac{2}{\sqrt{N^{2}+N}} Q_{N}(\{f, g\})+O\left(\frac{4}{N^{2}+N}\right),} \\
\frac{1}{2 \pi} \int_{S^{2 \mid 2}} d \mu_{S^{2 \mid 2}} f=-\operatorname{STr}\left(Q_{N}(f)\right)+O\left(\frac{2}{\sqrt{N^{2}+N}}\right) .
\end{gathered}
$$

Obviously, the parameter $2 / \sqrt{N^{2}+N}$ plays the role of the Planck constant for the quantization map $Q_{N}$. It is also important to stress that STr stands for the supertrace of the $(N+1 \mid N) \times(N+1 \mid N)$ supermatrices while, throughout this paper, we reserve the symbol str for the supertrace of $(2 \mid 1) \times$ (2|1) supermatrices.

To give a flavor, what the map $Q_{N}$ is about, let us make explicit the quantized versions of the functions $1, w, y_{k}, \theta, \bar{\theta}, \zeta$, and $\bar{\zeta}$ on $S^{2 \mid 2}$,

$$
Q_{N}(1)=\left(\begin{array}{cc}
\mathbf{1}_{N+1} & 0 \\
0 & \mathbf{1}_{N}
\end{array}\right), \quad Q_{N}(w)=\left(\begin{array}{cc}
-\sqrt{\frac{N}{N+1}} \mathbf{1}_{N+1} & 0 \\
0 & -\sqrt{\frac{N+1}{N}} \mathbf{1}_{N}
\end{array}\right),
$$




$$
\begin{gathered}
Q_{N}\left(y_{k}\right)=\left(\begin{array}{cc}
\sqrt{\frac{N+2}{N+1}} Q_{N+1}\left(x_{k}\right) & 0 \\
0 & \sqrt{\frac{N-1}{N}} Q_{N}\left(x_{k}\right)
\end{array}\right), \\
Q_{N}(\theta)=\frac{1}{\sqrt{N^{2}+N}}\left(\begin{array}{cc}
0 & T_{1} \\
T_{2}^{\dagger} & 0
\end{array}\right), \quad Q_{N}(\bar{\theta})=\frac{1}{\sqrt{N^{2}+N}}\left(\begin{array}{cc}
0 & T_{2} \\
-T_{1}^{\dagger} & 0
\end{array}\right), \\
Q_{N}(\zeta)=\frac{1}{\sqrt{N^{2}+N}}\left(\begin{array}{cc}
0 & -T_{2} \\
-T_{1}^{\dagger} & 0
\end{array}\right), \quad Q_{N}(\bar{\zeta})=\frac{1}{\sqrt{N^{2}+N}}\left(\begin{array}{cc}
0 & -T_{1} \\
T_{2}^{\dagger} & 0
\end{array}\right) .
\end{gathered}
$$

Here, $\mathbf{1}_{N}$ stands for the unit $N \times N$-matrix, $Q_{N}\left(x_{k}\right)$ are the quantized generators of the ordinary bosonic fuzzy sphere, and the $(N+1) \times N$ matrices $T_{1}, T_{2}$ are given by

$$
T_{1}:=\left(\begin{array}{cccc}
\sqrt{N} & 0 & \ldots & 0 \\
0 & \sqrt{N-1} & \ldots & 0 \\
0 & \ldots & \ldots & 0 \\
0 & \ldots & \ldots & 0 \\
0 & \ldots & \sqrt{2} & 0 \\
0 & \ldots & 0 & \sqrt{1} \\
0 & \ldots & 0 & 0
\end{array}\right), T_{2}:=\left(\begin{array}{cccc}
0 & 0 & \ldots & 0 \\
\sqrt{1} & 0 & \ldots & 0 \\
0 & \sqrt{2} & \ldots & 0 \\
0 & \ldots & \ldots & 0 \\
0 & \ldots & \ldots & 0 \\
0 & \ldots & \sqrt{N-1} & 0 \\
0 & \ldots & 0 & \sqrt{N}
\end{array}\right) .
$$

In particular, it is then easy to verify that it holds the basic fuzzy supersphere relation,

$$
Q_{N}\left(y_{1}\right)^{2}+Q_{N}\left(y_{2}\right)^{2}+Q_{N}\left(y_{3}\right)^{2}+Q_{N}(\theta) Q_{N}(\bar{\theta})-Q_{N}(\bar{\theta}) Q_{N}(\theta)=Q_{N}(1) .
$$

In what follows, we shall adopt a notation keeping the dependence on $N$ tacit,

$$
\hat{y}_{k}:=Q_{N}\left(y_{k}\right), \hat{w}:=Q_{N}(w), \hat{\theta}:=Q_{N}(\theta), \hat{\bar{\theta}}:=Q_{N}(\bar{\theta}), \hat{\zeta}:=Q_{N}(\zeta), \hat{\bar{\zeta}}:=Q_{N}(\bar{\zeta}) .
$$

It can be straightforwardly checked that the following supermatrices $L_{k}, V, \bar{V}$,

$$
L_{k}:=\frac{\sqrt{N^{2}+N}}{2} \hat{y}_{k}, \quad V:=\frac{\sqrt{N^{2}+N}}{2} Q_{N}(\theta), \quad \bar{V}:=\frac{\sqrt{N^{2}+N}}{2} Q_{N}(\bar{\theta}),
$$

realize a $(2 N+1)$-dimensional graded unitary representation of the Lie superalgebra $\operatorname{uosp}(2 \mid 1)$. The case $N=1$ corresponds to the defining representation of $\operatorname{uosp}(2 \mid 1)$ in terms of $(2 \mid 1) \times(2 \mid 1)$ supermatrices.

In what follows, we shall need the fuzzy versions of the supermatrices $\mathcal{M}$ and $\mathcal{K}$. We define them as

$$
\hat{\mathcal{M}}:=\left(\begin{array}{ccc}
\hat{y}_{3} & \hat{y}_{1}-\mathrm{i} \hat{y}_{2} & -\hat{\bar{\theta}} \\
\hat{y}_{1}+\mathrm{i} \hat{y}_{2} & -\hat{y}_{3} & \hat{\theta} \\
\hat{\theta} & \hat{\bar{\theta}} & 0
\end{array}\right), \quad \hat{\mathcal{K}}=\left(\begin{array}{ccc}
\hat{w} & 0 & \hat{\zeta} \\
0 & \hat{w} & -\hat{\bar{\zeta}} \\
\hat{\bar{\zeta}} & \hat{\zeta} & 2 \hat{w}
\end{array}\right) .
$$

Note that $\hat{\mathcal{M}}$ is of the $v$-type, while $\hat{\mathcal{K}}$ is of $s$-type. The supermatrices $\hat{\mathcal{M}}$ and $\hat{\mathcal{K}}$ turn out to fulfil the following identities which will be useful to show emergence of the supersymmetric Schwinger model (3.37) as the large $N$ limit of a certain supermatrix model. Here, they are

$$
\begin{gathered}
\hat{\mathcal{M}}^{2}=\frac{N+1 / 2}{\sqrt{N^{2}+N}} \hat{\mathcal{K}}+2-\frac{3 / 2}{\sqrt{N^{2}+N}} \hat{\mathcal{M}}, \\
(\hat{\mathcal{K}}+2)^{2}=(\hat{\mathcal{K}}+2)+\frac{1}{\sqrt{N^{2}+N}} \hat{\mathcal{M}}+\frac{3}{2}\left(1-\sqrt{\frac{N}{N+1}}\right)\left(1-\sqrt{\frac{N+1}{N}}\right) \hat{\mathcal{K}}, \\
\hat{\mathcal{M}} \hat{\mathcal{K}}=-\frac{N+1 / 2}{\sqrt{N^{2}+N}} \hat{\mathcal{M}}-\frac{1 / 2}{\sqrt{N^{2}+N}} \hat{\mathcal{K}} .
\end{gathered}
$$




\section{E. Supermatrix model}

Now we describe the construction of the manifestly supersymmetric gauge theory living on the fuzzy supersphere which in the large $N$ limit yields supersymmetric electrodynamics (3.37). The superfields present in this noncommutative theory are simply the $Q_{N}$-quantizations of the superfields $\Phi, C, \bar{C}$, and $C_{0}$ living on the ordinary supersphere and we shall denote them as $\hat{\Phi}, \hat{C}, \hat{\bar{C}}$, and $\hat{C}_{0}$. Thus, $\hat{\Phi}$ and $\hat{C}_{0}$ will be even $(N+1 \mid N) \times(N+1 \mid N)$ supermatrices $\left(\hat{C}_{0}\right.$ Hermitian $)$ and $\hat{C}, \hat{\bar{C}}$ will be odd $(N+1 \mid N) \times(N+1 \mid N)$ supermatrices Hermitian-conjugated to each other. As in the commutative case, we arrange the fuzzy gauge superfields $\hat{C}_{0}, \hat{C}$, and $\hat{\bar{C}}$ into the traceless Hermitian $(2 \mid 1) \times(2 \mid 1)$ supermatrix $\hat{C}$ of the $s$-type,

$$
\hat{C}:=\left(\begin{array}{ccc}
\hat{C}_{0} & 0 & \hat{C} \\
0 & \hat{C}_{0} & -\hat{\bar{C}} \\
\hat{\bar{C}} & \hat{C} & 2 \hat{C}_{0}
\end{array}\right) .
$$

We shall require, moreover, that $\hat{C}$ obey the following constraint:

$$
\operatorname{str}\left(\hat{\mathcal{K}} \hat{C}+\hat{C} \hat{\mathcal{K}}+\frac{2}{\sqrt{N^{2}+N}} \hat{C} \hat{C}\right)=0 .
$$

Note that constraint (3.83) is the fuzzy analogue of commutative constraint (3.23) because it follows from (3.67),

$$
\operatorname{str}\left(\hat{\mathcal{K}} \hat{C}+\hat{C} \hat{\mathcal{K}}+\frac{2}{\sqrt{N^{2}+N}} \hat{C} \hat{C}\right)=2 Q_{N}(\operatorname{str}(\mathcal{K} C))+O\left(\frac{2}{\sqrt{N^{2}+N}}\right) .
$$

Here, recall that str stands for the supertrace of $(2 \mid 1) \times(2 \mid 1)$ supermatrices, whereas the symbol $\mathrm{STr}$ (used, e.g., in the next equation) denotes the supertrace of the $(N+1 \mid N) \times(N+1 \mid N)$ supermatrices.

Consider now an action

$$
S_{N}(\hat{\Phi}, \mathcal{P})=-\frac{\pi\left(N^{2}+N\right)}{2} \operatorname{str} \operatorname{STr}\left((\mathcal{P} \hat{\Phi}-\hat{\Phi} \hat{\mathcal{K}})^{\dagger}(\mathcal{P} \hat{\Phi}-\hat{\Phi} \hat{\mathcal{K}})+\frac{1}{e^{2}}[\mathcal{P}, \mathcal{F}(\mathcal{P})]^{2}\right),
$$

where

$$
\mathcal{P}:=\hat{\mathcal{K}}+\frac{2}{\sqrt{N^{2}+N}} \hat{\mathcal{C}}, \quad \mathcal{F}(\mathcal{P}):=-\mathrm{i}\left(\frac{N^{2}+N}{4}\right)^{\frac{3}{2}} \operatorname{str}\left(\mathcal{P}_{v}^{2} \mathcal{P}_{v}^{2}-\hat{\mathcal{K}}_{v}^{2} \hat{\mathcal{K}}_{v}^{2}\right)
$$

and $\mathcal{P}_{v}^{2}$ means the $v$-type part of the supermatrix $\mathcal{P}^{2}$ in the sense of decomposition (3.4).

Eqs. (3.79) and (3.80) imply that the expression $\operatorname{str}\left(\hat{\mathcal{K}}_{v}^{2} \hat{\mathcal{K}}_{v}^{2}\right)$ commutes with any function on the fuzzy supersphere $S_{N}^{2 \mid 2}$. It hence follows that action (3.85) is invariant with respect to a supergauge symmetry

$$
\hat{\Phi} \rightarrow \mathcal{U} \hat{\Phi}, \quad \mathcal{P} \rightarrow \mathcal{U} \mathcal{P} \mathcal{U}^{\dagger}
$$

where $\mathcal{U}$ is an arbitrary even superunitary $(N+1 \mid N) \times(N+1 \mid N)$ supermatrix. In particular, the fuzzy scalar superfield strength $\mathcal{F}(\mathcal{P})$ transforms as

$$
\mathcal{F}(\mathcal{P}) \rightarrow \mathcal{U} \mathcal{F}(\mathcal{P}) \mathcal{U}^{\dagger}
$$

In terms of the fuzzy superfield $\hat{C}$, the supergauge transformation takes the following form:

$$
\hat{C} \rightarrow \mathcal{U} \hat{C} \mathcal{U}^{\dagger}-\frac{\sqrt{N^{2}+N}}{2}[\hat{\mathcal{K}}, \mathcal{U}] \mathcal{U}^{\dagger}
$$

It can be equally easily checked that constraint (3.83), which can be rewritten as

$$
\operatorname{str}\left(\mathcal{P}^{2}-\hat{\mathcal{K}}^{2}\right)=0,
$$

is also supergauge invariant. 
Now we study the $\operatorname{uosp}(2 \mid 1)$ supersymmetry of fuzzy action (3.85) with respect to the $\operatorname{uos} p(2 \mid 1)$ variations of the superfields $\hat{\Phi}$ and $\mathcal{P}$,

$$
\delta_{\mathcal{V}} \hat{\Phi}:=-\mathrm{i}\left[\mathcal{V}_{N}, \hat{\Phi}\right], \quad \delta_{\mathcal{V}} \mathcal{P}:=-\mathrm{i}\left[\mathcal{V}_{N} \otimes \mathbf{1}_{2 \mid 1}+\mathbf{1}_{N+1 \mid N} \otimes \mathcal{V}, \mathcal{P}\right] .
$$

Here, 1 stands for the unit supermatrix with the size indicated by the subscript, $\mathcal{V}$ is the element of $\operatorname{uosp}(2 \mid 1)$ viewed as the $v$-type traceless even Hermitian supermatrix of the size $(2 \mid 1) \times(2 \mid 1)$, and $\mathcal{V}_{N}$ is the Hermitian supermatrix which represents $\mathcal{V}$ in the $(N+1 \mid N)$ representation of $\operatorname{uos} p(2 \mid 1)$ described in (3.77).

Restricting a Hermitian supermatrix to its $v$-part is an operation interchangeable with the $\operatorname{uosp}(2 \mid 1)$ transformation; hence, the supermatrix $\mathcal{P}_{v}^{2}$ transforms as

$$
\delta_{\mathcal{V}} \mathcal{P}_{v}^{2}=-\mathrm{i}\left[\mathcal{V}_{N} \otimes \mathbf{1}_{2 \mid 1}+\mathbf{1}_{N+1 \mid N} \otimes \mathcal{V}, \mathcal{P}_{v}^{2}\right]
$$

and $\mathcal{F}(\mathcal{P})$ transforms as

$$
\delta_{\mathcal{V}} \mathcal{F}(\mathcal{P})=-\mathrm{i}\left[\mathcal{V}_{N}, \mathcal{F}(\mathcal{P})\right] .
$$

The $\operatorname{uosp}(2 \mid 1)$ supersymmetry of action (3.85) now follows easily from (3.91) and (3.93), from the cyclic properties of the supertraces str and $\mathrm{STr}$, and from the fact that

$$
\left[\mathcal{V}_{N} \otimes \mathbf{1}_{2 \mid 1}+\mathbf{1}_{N+1 \mid N} \otimes \mathcal{V}, \mathcal{K}\right]=0
$$

The last thing to be done is to show that the large $N$ limit of supermatrix model action (3.85) gives action (3.37) of the supersymmetric electrodynamics on the (graded)commutative supersphere $S^{2 \mid 2}$. We start by evaluating explicitly the $v$-part of the matrix $\hat{C} \hat{\mathcal{K}}+\hat{\mathcal{K}} \hat{C}$,

$$
(\hat{C} \hat{\mathcal{K}}+\hat{\mathcal{K}} \hat{C})_{v}=\frac{1}{2}\left(\begin{array}{ccc}
{[\hat{\zeta}, \hat{\bar{C}}]+[\hat{\bar{\zeta}}, \hat{C}]} & 2[\hat{\zeta}, \hat{C}] & {\left[\hat{\zeta}, \hat{C}_{0}\right]-[\hat{w}, \hat{C}]} \\
*-2[\hat{\bar{\zeta}}, \hat{\bar{C}}] & -[\hat{\zeta}, \hat{\bar{C}}]-[\hat{\bar{\zeta}}, \hat{C}] & {[\hat{w}, \hat{\bar{C}}]-\left[\hat{\bar{\zeta}}, \hat{C}_{0}\right]} \\
*[\hat{w}, \hat{\bar{C}}]-\left[\hat{\bar{\zeta}}, \hat{C}_{0}\right] & {[\hat{w}, \hat{C}]-\left[\hat{\zeta}, \hat{C}_{0}\right]} & 0
\end{array}\right) .
$$

It is important to stress that all commutators appearing in (3.95) are graded. Since the commutator in (3.68) is also graded, we find from (3.68) that

$$
(\hat{C} \hat{\mathcal{K}}+\hat{\mathcal{K}} \hat{C})_{v}=\frac{\mathrm{i}}{\sqrt{N^{2}+N}} Q_{N}(\{C, \mathcal{K}\}+\{\mathcal{K}, C\})+O\left(\frac{4}{N^{2}+N}\right) .
$$

From formula (3.80), we deduce

$$
\left(\hat{\mathcal{K}}^{2}\right)_{v}=\frac{1}{\sqrt{N^{2}+N}} \hat{\mathcal{M}} .
$$

This fact and formula (3.96) allow us to find the expansion of $\left(\mathscr{P}^{2}\right)_{v}$ in the Planckian constant $2 / \sqrt{N^{2}+N}$,

$$
\left(\mathcal{P}^{2}\right)_{v}=\frac{1}{\sqrt{N^{2}+N}} \hat{\mathcal{M}}+\frac{2 \mathrm{i}}{N^{2}+N} Q_{N}(\{C, \mathcal{K}\}+\{\mathcal{K}, \mathcal{C}\})+O\left(\left(\frac{4}{N^{2}+N}\right)^{\frac{3}{2}}\right) .
$$

By using (3.98) and (3.86), we immediately infer the expansion of the fuzzy superfield strength $\mathcal{F}(\mathcal{P})$ in the Planckian constant,

$$
\begin{gathered}
\mathcal{F}(\mathcal{P})=\frac{\sqrt{N^{2}+N}}{4 \mathrm{i}} \operatorname{str}\left((\hat{\mathcal{C}} \hat{\mathcal{K}}+\hat{\mathcal{K}} \hat{\mathcal{C}})_{v} \hat{\mathcal{M}}+\hat{\mathcal{M}}(\hat{C} \hat{\mathcal{K}}+\hat{\mathcal{K}} \hat{C})_{v}\right)+O\left(\frac{2}{\sqrt{N^{2}+N}}\right)= \\
=\frac{1}{2} Q_{N}(\operatorname{str}(\mathcal{M}\{\mathcal{K}, C\}+\{C, \mathcal{K}\} \mathcal{M}))+O\left(\frac{2}{\sqrt{N^{2}+N}}\right) .
\end{gathered}
$$

Then, we find from (3.26), (3.68), (3.69), and from the first equation of (3.86) that the full kinetic term in fuzzy action (3.85) expands as 


$$
\begin{gathered}
\frac{-\pi\left(N^{2}+N\right)}{2 e^{2}} \operatorname{strS} \operatorname{Tr}[\mathcal{P}, \mathcal{F}(\mathcal{P})]^{2}=\frac{2 \pi}{e^{2}} \operatorname{STr} Q_{N}\left(\operatorname{str}\{\mathcal{K}, \mathcal{F}(C)\}^{2}\right)+O\left(\frac{2}{\sqrt{N^{2}+N}}\right)= \\
=-\frac{1}{e^{2}} \operatorname{str} \int d \mu_{S^{2 \mid 2}}\{\mathcal{K}, \mathcal{F}(C)\}^{2}+O\left(\frac{2}{\sqrt{N^{2}+N}}\right) .
\end{gathered}
$$

In this way, we have recovered from the kinetic term of the fuzzy action in the large $N$ limit the kinetic term of (graded)commutative action (3.37).

The calculation of the large $N$ limit of the matter kinetic term in (3.85) is much easier. In fact, the immediate application of (3.68), (3.69), and of the first equation of (3.86) yields

$$
-\mathrm{i} \frac{\sqrt{N^{2}+N}}{2}(\mathcal{P} \hat{\Phi}-\hat{\Phi} \hat{\mathcal{K}})=Q_{N}(\{\mathcal{K}, \Phi\}-\mathrm{i} C \Phi)+O\left(\frac{2}{\sqrt{N^{2}+N}}\right) .
$$

Finally, putting together (3.100) and (3.101) and exploiting (3.69), we conclude that the large $N$ limit of fuzzy action (3.85) is action (3.37) of the supersymmetric electrodynamics on the (graded)commutative supersphere. Moreover, it can be obtained from (3.68) and (3.69) that the gauge symmetry and the $\operatorname{uos} p(2 \mid 1)$ supersymmetry of the fuzzy action induce in the $N \rightarrow \infty$ limit the gauge symmetry and the $\operatorname{uos} p(2 \mid 1)$ supersymmetry of (graded)commutative action (3.37).

\section{DISCUSSION OF THE RESULTS AND OUTLOOK}

The reader might have noticed that in the (graded)commutative part of our work, the matter superfield $\Phi$ was viewed just as the complex superfunction on the supersphere $S^{2 / 2}$ and not as a section of a nontrivial line bundle over $S^{2 \mid 2}$. Said in other words, we did not yet include supervortices in the formalism. From the physical point of view, such inclusion is necessary since the topologically nontrivial configurations usually play an important role in the quantum dynamics of electromagnetically interacting matter in two dimensions. Of course, the problem may be circumvented by studying just vortices and not supervortices. This means, in other words, to expand the manifestly supersymmetric action of the Schwinger models in components and to promote the complex scalar boson $\phi$ contained in the superfield $\Phi$ to a section of an appropriate line bundle. From the mathematical point of view, however, such a procedure is not very elegant and the inclusion of supervortices in a manifestly supersymmetric way represents actually an intriguing challenge.

The crucial point to understand is the geometrical status of the multiplet $C_{0}, C, \bar{C}$ of the gauge superfields. At the first sight, it looks natural to view $C_{0}, C, \bar{C}$ as constituent fields of some connection $C_{\text {; }}$ however, this hypothetical connection must have more constituents than just three superfields $C_{0}, C, \bar{C}$ because there are in total four independent directions on the supersphere (two even and two odd). The problem is that it is not a priori clear how to define covariant derivatives in all independent directions without introducing new dynamical fields into the action. To say the same thing more geometrically, it is not evident how to complete a partial connection (given by the covariant derivatives in the directions of the Hamiltonian vector fields $\{\zeta,\},.\{\bar{\zeta},$.$\} , and \{w,$.$\} ) into a full$ connection $C$. The usual trick which works well in the flat space expresses the covariant derivatives in even directions in terms of the anticommutators of the covariant derivatives in odd directions. However, this method turns out not to work in the curved space. Indeed, we have checked that there is an obstruction to complete the partial connections $C_{0}, C, \bar{C}$ to a full connection $C_{\text {in }}$ in that particular way and, astonishingly enough, that this obstruction can be quantitatively expressed in terms of the scalar gauge superfield strength $\mathcal{F}(C)$ given by (3.26). That means, in other words, that only those partial connections $C_{0}, C, \bar{C}$ which have vanishing field strength $\mathcal{F}(C)$ can be extended to a full connection $\mathbf{C}$.

We believe that, at the present stage, it is wise to postpone the issue of the inclusion of the supervortices into the formalism and to concentrate beforehand onto two other clues capable to shed additional light on the problem. The first clue to follow is noncommutative. As argued by Steinacker in Ref. 33, the study of gauge theories on the noncommutative spaces can be simpler than on the commutatives ones. In particular, a lot of geometrically involved concepts like nontrivial fiber bundles, connections, and monopole sectors need not be introduced formerly but they arise 
simply and naturally from the noncommutative formalism. ${ }^{33}$ We expect that the generalisation of Steinacker's approach to the noncommutative supersymmetric setting may help to contribute to give a sound geometrical meaning to the partial connection fields $C_{0}, C, \bar{C}$. The second clue consists in closely examining the mathematical structure of gauge theories on the sphere with the extended $N=(2,2)$ supersymmetry and to inspect the geometrical status of their $N=(1,1)$ contents.

Needless to say, another problem awaiting a solution consists in calculating a partition function and related dynamical characteristics of supermatrix model (3.85) that we have constructed. Whether the fashionable method of localisation can be useful in this context is an open question.

${ }^{1}$ Balachandran, A. P. and Vaidya, S., "Instantons and chiral anomaly in fuzzy physics,” Int. J. Mod. Phys. A 16, 17 (2001), e-print arXiv:hep-th/9910129.

${ }^{2}$ Benini, F. and Cremonesi, S., "Partition functions of $N=(2,2)$ gauge theories on $S^{2}$ and vortices," Commun. Math. Phys. 334, 3, 1483 (2015); e-print arXiv:1206.2356 [hep-th].

${ }^{3}$ Benini, F., Park, D. S., and Zhao, P., "Cluster algebras from dualities of $2 d N=(2,2)$ quiver gauge theories," Commun. Math. Phys. 340, 47-104 (2015); e-print arXiv:1406.2699 [hep-th].

${ }^{4}$ Carow-Watamura, U. and Watamura, S., "Noncommutative geometry and gauge theory on fuzzy sphere," Commun. Math. Phys. 212, 395 (2000); e-print arXiv:hep-th/9801195.

${ }^{5}$ Castellani, L., Catenacci, R., and Grassi, P. A., "The geometry of supermanifolds and new supersymmetric actions," Nucl. Phys. B 899, 112-148 (2015); e-print arXiv:1503.07886 [hep-th].

${ }^{6}$ Closset, C. and Cremonesi, S., "Comments on $N=(2,2)$ supersymmetry on two-manifolds," J. High Energy Phys. 1407, 75 (2014); e-print arXiv:1404.2636 [hep-th].

${ }^{7}$ Doroud, N. and Gomis, J., "Gauge theory dynamics and Kähler potential for Calabi-Yau complex moduli,” J. High Energy Phys. 1312, 99 (2013); e-print arXiv:1309.2305 [hep-th].

${ }^{8}$ Doroud, N., Gomis, J., Le Floch, B., and Lee, S., "Exact results in $D=2$ supersymmetric gauge theories," J. High Energy Phys. 1305, 93 (2013); e-print arXiv:1206.2606 [hep-th].

${ }^{9}$ Ferrara, S., "Supersymmetric gauge theories in two dimensions," Lett. Nuovo Cimento 13, 629 (1975).

${ }^{10}$ Festuccia, G. and Seiberg, N., "Rigid supersymmetric theories in curved superspace," J. High Energy Phys. 1106, 114 (2011).

${ }^{11}$ Gerchkovitz, E., Gomis, J., and Komargodski, Z., "Sphere partition functions and the Zamolodchikov metric," J. High Energy Phys. 1411, 1 (2014); e-print arXiv:1405.7271 [hep-th].

${ }^{12}$ Gomis, J. and Floch, B. L., "M2-brane surface operators and gauge theory dualities in Toda," e-print arXiv:1407.1852 [hep-th].

${ }^{13}$ Gomis, J. and Lee, S., "Exact Kähler potential from gauge theory and mirror symmetry," J. High Energy Phys. 1304, 19 (2013); e-print arXiv: 1210.6022 [hep-th]

14 Grosse, H., Klimčík, C., and Prešnajder, P., "Field theory on a supersymmetric lattice," Commun. Math. Phys. 185, 155 (1997); e-print arXiv:hep-th/9507074.

${ }^{15}$ Hoppe, J., "Quantum theory of a massless relativistic surface and a two dimensional bound state problem," Ph.D. thesis, MIT, 1982), available at http://dspace.mit.edu/handle/1721.1/15717.

${ }^{16}$ Hori, K. and Romo, M., "Exact results in two-dimensional $(2,2)$ supersymmetric gauge theories with boundary," e-print arXiv:1308.2438 [hep-th].

${ }^{17}$ Hosomichi, K. and Lee, S., "Self-dual strings and 2D SYM," J. High Energy Phys. 1501, 76 (2015); e-print arXiv:1406. 1802 [hep-th]

${ }^{18}$ Iso, S. and Umetsu, H., "Gauge theory on noncommutative supersphere from supermatrix model," Phys. Rev. D 69, 105003 (2004); e-print arXiv:hep-th/0311005.

${ }^{19}$ Iso, S. and Umetsu, H., "Note on gauge theory on fuzzy supersphere," Phys. Rev. D 69, 105014 (2004), e-print arXiv:hep-th/ 0312307.

${ }^{20}$ Iso, S., Kimura, Y., Tanaka, K., and Wakatsuki, K., "Noncommutative gauge theory on fuzzy sphere from matrix model," Nucl. Phys. B 604, 121-147 (2001); e-print arXiv:hep-th/0101102.

${ }^{21}$ Jayewardena, C., "Schwinger model on $S^{2}$," Helvetica Physica Acta 61, 636 (1988).

${ }^{22}$ Karabali, D., Nair, V. P., and Polychronakos, A. P., "Spectrum of Schrodinger field in a noncommutative magnetic monopole," Nucl. Phys. B 627, 565-579 (2002); e-print arXiv:hep-th/0111249.

${ }^{23}$ Kimura, Y., "Noncommutative gauge theories on fuzzy sphere and fuzzy torus from matrix model," Prog. Theor. Phys. 106, 445 (2001); e-print arXiv:hep-th/0103192.

${ }^{24}$ Klimčík, C., "Gauge theories on the noncommutative sphere," Commun. Math. Phys. 199, 257 (1998); e-print arXiv:hep-th/ 9710153.

${ }^{25}$ Klimčík, C., "A nonperturbative regularization of the supersymmetric Schwinger model," Commun. Math. Phys. 206, 567 (1999); e-print arXiv:hep-th/9903112.

${ }^{26}$ Klimčík, C., "On Poisson geometry and supersymmetric sigma models," Mod. Phys. Lett. A 27, 1250216 (2012); e-print arXiv:1204.4654 [hep-th].

${ }^{27}$ Madore, J., "The fuzzy sphere," Classical Quantum Gravity 9, 69 (1992).

${ }^{28}$ Pestun, V., "Localization of gauge theory on a four-sphere and supersymmetric Wilson loops," Commun. Math. Phys. 313, 71 (2012); e-print arXiv:0712.2824 [hep-th].

${ }^{29}$ Rittenberg, V. and Scheunert, V., "Elementary construction of graded Lie groups," J. Math. Phys. 19, 709 (1978).

${ }^{30}$ Samsonov, I. B. and Sorokin, D., "Gauge and matter superfield theories on $S^{2}$," J. High Energy Phys. 1409, 97 (2014); e-print arXiv:1407.6270 [hep-th].

${ }^{31}$ Schwinger, J., "Gauge invariance and mass. II,” Phys. Rev. 128, 2425 (1962).

${ }^{32}$ Sharpe, E., "A few recent developments in $2 d(2,2)$ and $(0,2)$ theories," e-print arXiv:1501.01628 [hep-th]. 
${ }^{33}$ Steinacker, H., "Quantized gauge theory on the fuzzy sphere as random matrix model,” Nucl. Phys. B 679, 66 (2004); e-print arXiv:hep-th/0307075.

${ }^{34}$ Steinacker, H. and Szabo, R. J., "Nonabelian localization for gauge theory on the fuzzy sphere," J. Phys.: Conf. Ser. 103, 012017 (2008); e-print arXiv:0708.4365 [hep-th].

${ }^{35}$ Sugishita, S. and Terashima, S., "Exact results in supersymmetric field theories on manifolds with boundaries," J. High Energy Phys. 1311, 21 (2013); e-print arXiv:1308.1973 [hep-th].

${ }^{36}$ Ydri, B., "Notes on noncommutative supersymmetric gauge theory on the fuzzy supersphere," Int. J. Mod. Phys. A 22, 5179 (2007); e-print arXiv:0708.3065 [hep-th].

${ }^{37}$ This Kirillov-Costant-Souriau Poisson bracket is constructed in the standard way by viewing the supersphere as the coadjoint orbit of the supergroup $\operatorname{UOS} p(2 \mid 1)$.

${ }^{38}$ Of course, some work is needed to recover (2.1) from (2.3) which we leave to the reader. To do that, the most straightforward way is to view $S^{2}$ as the Riemann sphere and to use the standard complex coordinate $z$ to parametrize the complement of the north pole.

${ }^{39}$ The sphere $S^{2}$ can be viewed as the coadjoint orbit of the group $S O(3)$.

${ }^{40} \mathrm{~A}$ traceless Hermitian supermatrix $A$ is of the $v$-type if $A_{v}=A$ or, equivalently, if $A_{s}=0$. 